\title{
DOMINANT REPRESENTING MEASURES AND RATIONAL APPROXIMATION
}

\author{
BY \\ I. GLICKSBERG $\left({ }^{1}\right)$
}

1. Let $K$ be a compact set in the complex plane $C$. In 1951 Mergelyan proved that if $C \backslash K$ is connected, any continuous function on $K$ analytic on the interior $K^{0}$ of $K$ is uniformly approximable by polynomials in $z$. More generally, he later showed that if $\boldsymbol{C} \backslash K$ has finitely many components, such functions are uniformly approximable by rational functions $[\mathrm{M}]$.

Following a functional analytic approach due to Bishop [B 3] of comparing the spaces of measures orthogonal to the two function algebras involved, the first of these results was derived in [GIW] via results valid for dirichlet algebras from a classical result of Walsh [Wa 1] and a well-known result of Bishop [B 2]. Since not only dirichlet algebras are involved in the second result of Mergelyan, the same approach was not then applicable.

Recently, however, one of the dirichlet algebra results used in [GlW], the abstract F. and M. Riesz theorem, was extended to its proper setting by P. R. Ahern [A]; this provided the origin for the present paper, which began as an attempt to obtain the more general Mergelyan result by the approach of [GlW]. In the special case in which the components of $C \backslash K$ have disjoint closures this is easily done $\left(^{2}\right)$, but in general this requires answering some related questions, and leads to the interesting question of determining the dimension of the space of real measures on $\partial K$ orthogonal to all rational functions with poles off $K$. The answer to this question has quite recently been obtained by P. R. Ahern and Donald Sarason [AS 2] as a consequence of their earlier study of $H^{p}$ spaces for hypo-dirichlet (and slightly more general) algebras [AS 1], which had already given the Mergelyan result.

The main portion of this paper, $\S 3$, is devoted to an alternative proof of this result of Ahern and Sarason, and some extensions. We shall make use of two results of [AS 2] (Lemmas 1-3), both facts about harmonic measures resulting from the topology, independent of [AS 1] and also easily accessible; in general, however, our approach is quite different, depending more on the actual setting in the complex plane, and somewhat less on the global finite dimensionality of the real orthogonal measures implicit in the hypo-dirichlet situation, so that we obtain

Received by the editors September 29, 1966.

(1) Work supported in part by the National Science Foundation.

$\left({ }^{2}\right)$ This and the preliminary results of this paper were announced in [G1 2]; since the paper was completed many of the results of $\$ 2$ have been extended considerably in [G1 3], [GaGl]. 
some extensions to the case in which $C \backslash K$ has infinitely many components. These are very special, however, and in particular, our strongest results give more a means of constructing such $K$ with the continuous analytic functions on $K$ approximable by rational functions than a delineation of a specific class of sets for which such rational approximation holds (cf. (3.14), (3.15), and (3.19)).

$\$ 2$ is devoted to the central notion of dominant representing measures, and gives an alternate proof of Ahern's F. and M. Riesz theorem and an extension of a result of Hoffman and Wermer crucial for all that follows, along with various corollaries. $\S 4$ is concerned with the application of results of the preceding section to interpolation, while the final section gives an illustration of the use of various preparatory results in obtaining approximation within the uniform closure on $K$ of the rational functions with poles off $K$.

The author is indebted to Maynard G. Arsove for suggesting the proof of a key fact (Lemma 3.9) concerning harmonic measures, to John Wermer for pointing out some early errors, and to G. and L. Lumer, J. Ryff and W. B. Woolf for several helpful conversations. Our debt to [AS 2] will be clear.

In what follows $f \mu$ will denote the usual product of a function $f$ and a measure $\mu$, and, when convenient, $\mu(f)$ the integral $\int f d \mu$. For two nonnegative measures $\mu$ and $\lambda, \mu \ll \lambda$ will denote the absolute continuity of $\mu$ with respect to $\lambda$. For a set $F$, $\mu_{F}$ is the restriction of $\mu$ to $F$, and $\chi_{F}$ is its characteristic function.

Whenever a function algebra $A$ is being viewed as a subalgebra of $C(X)$ for a particular $X, A^{\perp}$ will denote the space of complex measures on that $X$ orthogonal to $A$.

Finally, for want of a better term, we shall call a bounded domain $U$ in $C$ nicely connected if it is simply connected and the (nontangential) boundary value extension of the Riemann map of $|z|<1$ onto $U$ is $1-1$ on all but a subset of measure zero of the unit circle.

2. Dominant representing measures. Let $X$ be a compact Hausdorff space, and $A$ a closed separating subalgebra of $C(X)$ containing the constants. For $\phi \in \mathfrak{M}=\mathfrak{M}_{A}$, the space of multiplicative linear functionals on $A$ (or spectrum of $A$ ), let $M_{\phi}=M_{\phi}(A)$ denote the (weak* compact, convex) set of all probability measures $\lambda$ on $X$ representing $\phi$ :

$$
\phi(f)=\int f d \lambda=\lambda(f), \quad f \in A .
$$

$M_{\phi}$ is nonvoid [We 3], and as a consequence $\operatorname{Re} \phi(f)=\int \operatorname{Re} f d \lambda$ is an (order preserving) function of $\operatorname{Re} f$ alone. This allows us to state the following (essentially familiar) consequence of the Hahn-Banach and Riesz representation theorems, which will be fundamental to our arguments.

Lemma 2.1. For $u \in C^{R}(X)$,

$$
\sup \{\operatorname{Re} \phi(f): f \in A, \operatorname{Re} f \leqq u\}=\inf \left\{\lambda(u): \lambda \in M_{\phi}\right\} .
$$


Proof. With $f$ and $u$ as above, $\operatorname{Re} \phi(f)=\int \operatorname{Re} f d \lambda \leqq \int u d \lambda$ for any $\lambda$ in $M_{\phi}$, so the left side of (2.1) is bounded by the right. On the other hand, $\operatorname{Re} f \rightarrow \operatorname{Re} \phi(f)$ is a nonnegative linear functional, and by a version [D] of the Hahn-Banach theorem has an order-preserving extension to $C^{R}(X)$ with precisely the left side of (2.1) as its value at $u$.

But this extension is represented by a nonnegative measure $\lambda$ on $X$, which must be a probability measure representing $\phi$ on $A$ since $\lambda(\operatorname{Re} f)=\operatorname{Re} \phi(f), f \in A$, and this implies $\lambda(1)=1$ and $\lambda(f)=\phi(f), f \in A$. So $\lambda \in M_{\phi}$ and $\lambda(u)$ is precisely the left side of (2.1), showing (2.1) holds.

DEFINITION 2.2. We call $\lambda \in M_{\phi}$ dominant (or a dominant representing measure for $\phi)$ if $M_{\phi} \subset L_{1}(\lambda) ; \lambda$ is strongly dominant if there is a constant $k$ for which

$$
\lambda^{\prime} \leqq k \lambda, \text { all } \lambda^{\prime} \in M_{\phi} .
$$

Ahern's extension of the abstract F. and M. Riesz theorem (previously known when $M_{\phi}$ was a singleton) is to the case in which $M_{\phi}$ has a dominant representing measure. His result is basic to what we shall do, and we shall give a proof of the result (Theorem 2.4) based on (2.1). First, however, some remarks on dominant representing measures are in order.

Remarks. (2.3a). A dominant representing measure for $M_{\phi}$ clearly exists when $M_{\phi}$ contains a norm dense sequence $\left\{\lambda_{n}\right\}$, e.g., $\sum_{1}^{\infty} 2^{-n} \lambda_{n}=\lambda$ is easily seen to be dominant. And when $X$ is metrizable $M_{\phi}$ must be norm separable if a dominant representing measure $\lambda$ exists since $L_{1}(\lambda)$ is then itself norm separable.

(2.3b). More generally, a dominant $\lambda$ exists whenever $M_{\phi}$ is contained in $L_{1}(\mu)$ for any measure $\mu$. This follows from a simple exhaustion argument, as follows . Let $\mu_{\lambda}$ be the absolutely continuous component of $\mu$ relative to $\lambda \in M_{\phi}$, and set $C=\sup \left\{\left\|\mu_{\lambda}\right\|: \lambda \in M_{\phi}\right\}$. Choose $\lambda_{n}$ so that $\left\|\mu_{\lambda_{k}}\right\| \rightarrow C$, and then set $\lambda=\sum_{1}^{\infty} 2^{-n} \lambda_{n}$, so clearly $\left\|\mu_{\lambda}\right\|=C$. If $M_{\phi} \Phi L_{1}(\lambda)$ we have a $\lambda^{\prime}$ in $M_{\phi}$ and $E \subset X$ with $\lambda(E)=0$, $\lambda^{\prime}(E)>0$ whence $\mu(E)>0$ and clearly $\left\|\mu_{\left(\lambda+\lambda^{\prime}\right) / 2}\right\|>C$, our contradiction.

(2.3c). The difference of two elements of $M_{\phi}$ is a real measure orthogonal to $A$, and thus if the space of such real measures is norm separable, dominant representing measures exist for every $\phi \in \mathfrak{M}$. In particular, this is the case if $(\operatorname{Re} A)^{\perp}$, which amounts to the complex span of the real measures on $X$ orthogonal to $A$, is finite dimensional. And in that case, strongly dominant measures exist. In fact, a strongly dominant $\lambda \in M_{\phi}$ exists whenever $M_{\phi}$ can be realized as a bounded convex body in some normed space. For if $\lambda$ is interior to $M_{\phi}$ in that realization, since $M_{\phi}$ is bounded one has $\lambda+\varepsilon\left(\lambda-M_{\phi}\right) \subset M_{\phi}$ for a sufficiently small $\varepsilon>0$, whence $(1+\varepsilon) \lambda$ $-\varepsilon \lambda^{\prime} \geqq 0$ for all $\lambda^{\prime}$ in $M_{\phi}$.

(2.4). The principal consequence of the existence of a dominant representing measure is Ahern's F. and M. Riesz theorem [A], which follows from the following lemma, inspired in part by the approach of Forelli $[\mathrm{F}]$.

LEMMA 2.5 (AHERN [A]). Suppose $\lambda$ is a dominant representing measure for $\phi$, 
and $E$ is a $\sigma$-compact set of $\lambda$-measure zero. Then there is a sequence $\left\{g_{n}\right\}$ in the unit ball of $A$ with $g_{n} \rightarrow 0$ pointwise on $E$ and $g_{n} \rightarrow 1$ a.e. $\lambda$.

Proof. Let $K$ be a compact subset of $E$, and $n$ a positive integer. Because $K$ is compact and $\lambda$ is regular, and $\lambda K=0$, we can find a sequence $\left\{u_{k}\right\}$ in $C^{R}(X)$ with

(i) $-n \leqq u_{k} \leqq 0$,

(ii) $u_{k}=-n$ on $K$,

(iii) $\lambda\left(u_{k}\right) \rightarrow 0$.

Indeed we can clearly take $\left\{u_{k}\right\}$ nondecreasing, replacing $u_{k}$ by $\sup \left\{u_{j}: j \leqq k\right\}$.

Since $\left\{u_{k}\right\}$ is monotonic, (iii) implies $u_{k} \rightarrow 0$ a.e. $\lambda$, and since $\lambda$ is dominant, $\lambda^{\prime}\left(u_{k}\right) \rightarrow 0$ for each $\lambda^{\prime}$ in $M_{\phi}$ by monotone convergence. But now $f_{k}\left(\lambda^{\prime}\right)=\lambda^{\prime}\left(u_{k}\right)$ defines a sequence $\left\{f_{k}\right\}$ in $C\left(M_{\phi}\right)$, where $M_{\phi}$ is given the weak* topology, and $f_{k} \nearrow 0$ pointwise on the compact space $M_{\phi}$. By Dini's theorem convergence is uniform, and for $k$ sufficiently large

$$
-1 / n<\inf \left\{\lambda^{\prime}\left(u_{k}\right): \lambda^{\prime} \in M_{\phi}\right\}
$$

and by (2.1) there is an $f$ in $A$ with

$$
\operatorname{Re} f \leqq u_{k} \leqq 0, \quad-1 / n<\operatorname{Re} \phi(f) .
$$

In particular $\operatorname{Re} f \leqq-n$ on $K$ by (ii), so that $\left|e^{f}\right|=e^{\mathrm{Re} f} \leqq 1$ everywhere and $\leqq e^{-n}$ on $K$, while $\left|\phi\left(e^{f}\right)\right|=\left|e^{\phi(f)}\right|=e^{\mathrm{Re} \phi(f)}>e^{-1 / n}$. With $\xi=\operatorname{sgn} \phi\left(e^{f}\right)$, we thus have an element $g=\xi e^{f}$ in the unit ball of $A$ with $1 \geqq \phi(g)>e^{-1 / n}$ and $|g| \leqq e^{-n}$ on $K$.

Now $E$ is the union of an increasing sequence $K_{n}$ of compacta, and applying the preceding argument to $n$ and $K=K_{n}$ we obtain a $g_{n}$ in the unit ball of $A$ with $\left|g_{n}\right| \leqq e^{-n}$ on $K_{n}$ (so $g_{n} \rightarrow 0$ on $E$ ) and $1 \geqq \phi\left(g_{n}\right)>e^{-1 / n}$. Since

$$
\begin{aligned}
0 & \leqq \int\left|1-g_{n}\right|^{2} d \lambda=1+\int\left|g_{n}\right|^{2} d \lambda-2 \operatorname{Re} \int g_{n} d \lambda \\
& =1+\int\left|g_{n}\right|^{2} d \lambda-2 \operatorname{Re} \phi\left(g_{n}\right) \leqq 1+1-2 \operatorname{Re} \phi\left(g_{n}\right) \rightarrow 0,
\end{aligned}
$$

we have $g_{n} \rightarrow 1$ in $L_{2}(\lambda)$; passing to a subsequence, $g_{n} \rightarrow 1$ a.e. $\lambda$, as desired, and our proof is complete.

As an immediate consequence of Lemma 2.5 we have Ahern's F. and M. Riesz theorem.

ThIOREM 2.6 [A]. Let $\mu \in A^{\perp}$ and let $\lambda$ be a dominant representing measure for $\phi \in \mathfrak{M}$. Let $\mu_{\lambda}$ and $\mu_{\lambda}^{\prime}$ be the absolutely continuous and singular components of $\mu$ with respect to $\lambda$. Then $\mu_{\lambda}$ and $\mu_{\lambda}^{\prime}$ are orthogonal to $A$.

For the proof we need only note that $\mu_{\lambda}^{\prime}$ is carried by a $\sigma$-compact set $E$ of $\lambda$ measure zero, so that if $\left\{g_{n}\right\}$ is the sequence in the unit ball of $A$ we obtain from (2.5) then $\left\|g_{n} \mu-\mu_{\lambda}\right\|=\left\|g_{n} \mu_{\lambda}+g_{n} \mu_{\lambda}^{\prime}-\mu_{\lambda}\right\| \rightarrow 0$ by dominated convergence; since $g_{n} \mu \in A^{\perp}$ for each $n, \mu_{\lambda} \in A^{\perp}$, whence $\mu_{\lambda}^{\prime}=\mu-\mu_{\lambda} \in A^{\perp}$. 
In [G] Gleason introduced the notion of a "part" of $\mathfrak{M}$ as an equivalence class in $\mathfrak{M}$ under the equivalence relation that $\phi_{1} \sim \phi_{2}$ if $\left\|\phi_{1}-\phi_{2}\right\|_{A^{*}}<2$ (where the

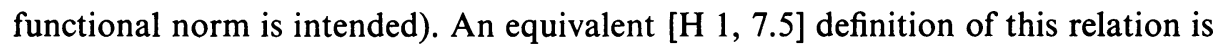
that $\phi_{1} \sim \phi_{2}$ iff $\hat{g}_{n}\left(\phi_{1}\right) \rightarrow 1$ whenever $\hat{g}_{n}\left(\phi_{2}\right) \rightarrow 1$, and vice versa, for any sequence $\left\{g_{n}\right\}$ in the unit ball of $A$. This yields the next consequence of 2.5 .

COROLlaRY 2.7. If $\phi$ has a dominant representing measure $\lambda$ then any $\phi_{1}$ in the same Gleason part has a dominant representing measure equivalent to (i.e., mutually absolutely continuous with) $\lambda$.

Let $\lambda_{1} \in M_{\phi_{1}}$ and let $E$ be a compact set with $\lambda(E)=0$. By 2.5 we have a sequence $\left\{g_{n}\right\}$ in the unit ball of $A$ with $g_{n} \rightarrow 0$ on $E$ and $g_{n} \rightarrow 1$ a.e. $\lambda$, so that $\hat{g}_{n}(\phi) \rightarrow 1$. Thus $\lambda_{1}\left(g_{n}\right)=\hat{g}_{n}\left(\phi_{1}\right) \rightarrow 1$, and since lim sup $\left|\lambda_{1}\left(g_{n}\right)\right| \leqq 1-\lambda_{1}(E)$, we conclude that $\lambda_{1}(E)=0$, and therefore that $\lambda_{1} \ll \lambda$. As noted in (2.3b), $M_{\phi_{1}} \subset L_{1}(\lambda)$ implies $\phi_{1}$ has a dominant representing measure $\lambda_{1}$, and of course $\lambda_{1} \ll \lambda$. By symmetry $\lambda \ll \lambda_{1}$, and we are done.

If $\phi_{1}, \phi_{2} \in \mathfrak{M}$ have representing measures $\lambda_{1}, \lambda_{2}$ which are not mutually singular, then $\left\|\phi_{1}-\phi_{2}\right\|_{A^{*}} \leqq\left\|\lambda_{1}-\lambda_{2}\right\|<2$, so that $\phi_{1} \sim \phi_{2}$. Thus any two dominant representing measures are either equivalent or mutually singular, depending on whether the corresponding homomorphisms are in the same or distinct parts.

Definitions. (1) $A$ measure $\sigma$ orthogonal to $A$ is completely singular if $|\sigma|$ and each representing measure are mutually singular.

(2) For convenience we call a part trivial if it consists of a peak point.

We of course have a dominant representing measure for such a point, but no nonzero orthogonal measures are absolutely continuous with respect to the dominant one, and for this reason trivial parts need not appear in our next result, which follows exactly as in [GIW] by virtue of 2.6.

THEOREM 2.8. Suppose each nontrivial part $P_{v}$ of $\mathfrak{M}$ contains an element with a dominant representing measure $\lambda_{v}$. Then for any $\mu$ in $A^{\perp}$ we have

$$
\mu=\sum_{v} \mu_{\lambda_{v}}+\sigma
$$

where $\sigma$ is a completely singular orthogonal measure, $\mu_{\lambda_{v}}$ is the absolutely continuous part of $\mu$ relative to $\lambda_{v}$, the summands on the right are mutually singular and orthogonal to $A$, and the (actually countable) sum converges in norm.

Setting $A_{\lambda_{v}}^{\perp}=\left\{\mu_{\lambda_{v}}: \mu \in A^{\perp}\right\}$, which coincides with $\left\{\mu \in A^{\perp}: \mu \ll \lambda_{v}\right\}$ by 2.6 , and can be viewed as a closed subspace of $L^{1}\left(\lambda_{v}\right)$, we can write (2.2) as

$$
A^{\perp}=\sum_{v} A_{\lambda_{v}}^{\perp}+S
$$

where $S$ is the closed space of completely singular orthogonal measures and the first summand represents all norm convergent series. In order to further identify 
the components $A_{\lambda}^{\frac{1}{\lambda}}$ we need strongly dominant representing measures (cf. (2.2)) to obtain the following extension of a result $\left({ }^{3}\right)$ of Hoffman and Wermer [We 5]. First we recall the

Definition. $H^{p}(\lambda)=H^{p}(A, \lambda)$ denotes the closure in $L^{p}(\lambda), p<\infty$, of $A$, and $H^{\infty}(\lambda)=L^{\infty}(\lambda) \cap H^{2}(\lambda)$. Finally $H_{0}^{p}(\lambda)=\left\{f \in H^{p}(\lambda): \int f d \lambda=0\right\}$.

THEOREM 2.9. Suppose $\lambda$ is a strongly dominant representing measure for $\phi$, and $h \in L^{\infty}(\lambda) \cap H^{2}(\lambda)=H^{\infty}(A)$. Then there is a sequence $\left\{f_{n}\right\}$ in $A$ with $\left\|f_{n}\right\| \leqq\|h\|_{\infty}$, which converges to $h$ a.e. $\lambda$.

Thus $h \in L^{\infty}(\lambda) \cap H^{2}(\lambda)$ implies $h$ lies in the weak ${ }^{*}$ closure of $A$ in $L^{\infty}(\lambda)$.

Proof (following [We 5]). Let $\left\{g_{n}\right\}$ be a sequence in $A$ converging to $h$ in $L^{2}(\lambda)$. Replacing $\left\{g_{n}\right\}$ by a subsequence we can assume $g_{n} \rightarrow h$ a.e. $\lambda$, and without loss of generality we can assume $\|h\|_{\infty}=1$.

Let $E_{n}=\left\{x \in X:\left|g_{n}(x)\right| \geqq 1\right\}$. We assert that $\int_{E_{n}} \log \left|g_{n}\right| d \lambda \rightarrow 0$. Indeed for $\varepsilon>0$ if $G_{n}=\left\{x \in X:\left|g_{n}(x)\right| \geqq 1+\varepsilon\right\}$ then $\lambda\left(G_{n}\right) \rightarrow 0$ and

$$
\begin{aligned}
\int_{G_{n}} \log \left|g_{n}\right| d \lambda & \leqq \int_{G_{n}}\left|g_{n}\right| d \lambda \leqq \int_{G_{n}}\left|g_{n}-h\right|+|h| d \lambda \\
& \leqq\left\|g_{n}-h\right\|_{2} \cdot 1+\lambda\left(G_{n}\right) \rightarrow 0
\end{aligned}
$$

So $\int_{G_{n}} \log \left|g_{n}\right| d \lambda<\varepsilon$ for $n$ large, and

$$
\int_{E_{n}} \log \left|g_{n}\right| d \lambda \leqq \int_{G_{n}}+\int_{E_{n} \mid G_{n}}<\varepsilon+\log (1+\varepsilon)
$$

since $\left|g_{n}\right|<1+\varepsilon$ off $G_{n}$. Our assertion is now clear.

Now $u_{n}=-\log ^{+}\left|g_{n}\right| \in C^{R}(X)$ vanishes off $E_{n}, u_{n} \leqq 0$, and $0 \leqq \varepsilon_{n}=-\int u_{n} d \lambda$ $=\int \log { }^{+}\left|g_{n}\right| d \lambda=\int_{E_{n}} \log \left|g_{n}\right| d \lambda \rightarrow 0$. Since $\lambda$ is strongly dominant $k \lambda \geqq \lambda^{\prime}$ for all $\lambda^{\prime}$ in $M_{\phi}$, whence

$$
0 \geqq \int u_{n} d \lambda^{\prime} \geqq \frac{1}{k} \int u_{n} d \lambda=-\frac{\varepsilon_{n}}{k},
$$

$u_{n}$ being nonpositive. So

$$
\inf \left\{\lambda^{\prime}\left(u_{n}\right): \lambda^{\prime} \in M_{\phi}\right\} \geqq-\varepsilon_{n} / k
$$

and by (2.1) we have an $h_{n}$ in $A$ with $\operatorname{Re} h_{n} \leqq u_{n} \leqq 0$, and $\operatorname{Re} \phi\left(h_{n}\right)>-\varepsilon_{n} / k-1 / n$. Thus $\left|e^{h_{n}}\right| \leqq 1$, and in fact $\left|g_{n} e^{h_{n}}\right|=\left|g_{n}\right| e^{\mathrm{Re} h_{n}} \leqq\left|g_{n}\right| e^{-\log +\left|g_{n}\right|} \leqq 1$ while $1 \geqq\left|\phi\left(e^{h_{n}}\right)\right|$ $=e^{\operatorname{Re} \phi\left(h_{n}\right)}>e^{-\varepsilon_{n} / k-1 / n}$ which tends to 1 as $n \rightarrow \infty$. So if we set

$$
h_{n}^{\prime}=e^{h_{n}} \cdot \overline{\operatorname{sgn}} \phi\left(e^{h_{n}}\right)
$$

we obtain a sequence $\left\{h_{n}^{\prime}\right\}$ in the unit ball of $A$ with $h_{n}^{\prime} \rightarrow 1$ a.e. $\lambda$, exactly as in the last part of the proof of 2.5 , and $\left|g_{n} h_{n}^{\prime}\right| \leqq 1$. Now we need only set $f_{n}=g_{n} h_{n}^{\prime}$ to get our sequence $\left\{f_{n}\right\}$ in $A$ with $\left\|f_{n}\right\| \leqq\|h\|_{\infty}, f_{n} \rightarrow h$ a.e. $\lambda$.

$\left({ }^{3}\right)$ The version of this result suggested (with no mention of strong dominance) in [Gl 2] is erroneous. 
Finally, by dominated convergence $f_{n} \rightarrow h$ weak* in $L^{\infty}(\lambda)$, completing our proof.

We can now identify $A_{\lambda}^{\perp}$ when $\lambda$ is strongly dominant. Recall first that $(\operatorname{Re} A)^{\perp}$, the measures on $X$ orthogonal to $\operatorname{Re} A$, is spanned by real measures, which, since $\operatorname{Re} A=\operatorname{Im} A$, are orthogonal to $A$. Thus $(\operatorname{Re} A)^{\perp}$ is just the complex span of the real measures in $A^{\perp}$. We again set $(\operatorname{Re} A)_{\lambda}^{\perp}=\left\{\mu_{\lambda}: \mu \in(\operatorname{Re} A)^{\perp}\right\}$, which is the span in $L_{1}(\lambda) \cdot \lambda$ of its real elements orthogonal to $A$.

COROLlaRY 2.10. If $\lambda$ is strongly dominant representing measure for $\phi$ then $A_{\lambda}^{\perp}$ is the closure in the measure norm of $(\operatorname{Re} A)_{\lambda}^{\perp}+\phi^{-1}(0) \cdot \lambda$. In particular, if $(\operatorname{Re} A)_{\lambda}^{\perp}$ is finite dimensional,

$$
A_{\lambda}^{\perp}=(\operatorname{Re} A)_{\lambda}^{\perp}+\left(\phi^{-1}(0) \cdot \lambda\right)^{-}=(\operatorname{Re} A)_{\lambda}^{\perp}+H_{0}^{1}(\lambda) \cdot \lambda .
$$

In order to prove 2.10 we need an observation on $L^{2}(\lambda)$. As is easily seen $\lambda$ is multiplicative on $H^{2}(\lambda)$ (by Schwartz, $(f, g) \rightarrow \int f g d \lambda$ extends continuously from $A \times A$ to $H^{2} \times A$ to $\left.H^{2} \times H^{2}\right)$; so we have $H_{0}^{2}(\lambda)=\left\{f \in H^{2}(\lambda): \int f d \lambda=0\right\}$ orthogonal in $L^{2}(\lambda)$ to

$$
\overline{H^{2}(\lambda)}: f \in H_{0}^{2}(\lambda), \quad g \in H^{2}(\lambda)
$$

imply $\int f g d \lambda=0$. Moreover a standard elementary argument shows $H_{0}^{p}(\lambda)$ is the closure of $\phi^{-1}(0)$ in $L^{p}(\lambda)$. Thus we have the orthogonal decomposition

$$
L^{2}(\lambda)=H^{2}(\lambda) \oplus \overline{H_{0}^{2}(\lambda)} \oplus E,
$$

where $E$ is orthogonal to

$$
A+\overline{\phi^{-1}(0)}=A+\bar{A},
$$

hence to $\operatorname{Re} A$ : so $E$ lies in the span of the real elements of $L^{2}(\lambda)$ orthogonal to $A$, and clearly coincides with that span. Now we may write, with obvious but clumsy notation

$$
L^{2}(\lambda)=H^{2}(\lambda) \oplus \overline{H_{0}^{2}(\lambda)} \oplus(\operatorname{Re} A)_{2}^{\frac{1}{2}} .
$$

To proceed to the proof of 2.10 , we have $A_{\lambda}^{\perp} \supset(\operatorname{Re} A)_{\lambda}^{1}+\phi^{-1}(0) \lambda$ clearly, so we need only see any $h$ in $L^{\infty}(\lambda)$ orthogonal to the smaller set is orthogonal to $A_{\lambda}^{\perp}$. But such an $h$ lies in $L^{2}(\lambda)$ and in terms of $L^{2}$ orthogonality $\bar{h}$ is orthogonal to

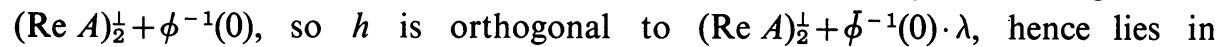
$H^{2}(\lambda)$ by (2.4). So by 2.9 we have $\left\{f_{n}\right\}$ in $A$ converging weak* to $h$ in $L^{\infty}(\lambda)$, and thus for any $\mu$ in $A_{\lambda}^{\perp} \subset L_{1}(\lambda) \cdot \lambda, 0=\int f_{n} d \mu \rightarrow \int h d \mu$, and $h \perp A_{\lambda}^{\perp}$ as desired.

For the final assertion of 2.10 we need only note that by [D, p. 14] $(\operatorname{Re} A)_{\lambda}^{\frac{1}{1}}$ $+\left(\phi^{-1}(0) \lambda\right)^{-}$is closed, while $H_{0}^{1}(\lambda)=\left(\phi^{-1}(0) \lambda\right)^{-}$as we noted earlier.

We shall want to apply the preceding results to the following question. Suppose we have two subalgebras $A \subset B$ of $C(X)$ for which $\mathfrak{M}_{A}=\mathfrak{M}_{B}$. Under what conditions can we assert $A=B$ ? We are of course limited to those situations in which dominant or strongly dominant representing measures exist, and in the actual cases we consider no completely singular measures exist. One case to which our results apply can be summed up as follows. 
Corollary 2.11. Suppose $A \subset B \subset C(X)$ have the same space $\mathfrak{M}$ of multiplicative linear functionals and $(\operatorname{Re} A)^{\perp}=(\operatorname{Re} B)^{\perp}$ is finite dimensional. Then if $A$ has no completely singular orthogonal measures, $A=B$.

We could alternatively assume the defects in $C^{R}(X)$ of $(\operatorname{Re} A)^{-}$and $(\operatorname{Re} B)^{-}$ be finite and equal, and more generally that the completely singular orthogonal measures for $A$ and $B$ simply coincide-but we have no application of this sort in mind. In order to see 2.11 note that for every multiplicative measure $\lambda_{1}$ on $A$ the corresponding homomorphism arises from one of $B$ by hypothesis, represented on $B \supset A$ by a measure $\lambda_{2}$ multiplicative on $B$. So $\lambda_{1}-\lambda_{2} \in(\operatorname{Re} A)^{\perp}=(\operatorname{Re} B)^{\perp}$, and $\lambda_{1}$ is multiplicative on $B$ as well. Thus the multiplicative measures for the two algebras coincide.

Since $(\operatorname{Re} A)^{\perp}=(\operatorname{Re} B)^{\perp}$ is finite dimensional, strongly dominant measures exist by (2.3c), and these clearly coincide for both algebras by their definition. Since parts in $\mathfrak{M}_{A}=\mathfrak{M}_{B}$ for each algebra are determined by the equivalence or mutual singularity of dominant representing measures ( 2.7 and below), the parts coincide for both algebras (a fact which can be seen in various other ways). Moreover, a completely singular measure orthogonal to $B$ is orthogonal to $A$, so must vanish. Thus, we have a collection $\left\{\lambda_{v}\right\}$ of strongly dominant representing measures, one for each part, and by Theorem 2.8 and (2.3) $A^{\perp}=\sum A_{\lambda_{v}}^{\perp}, B^{\perp}=\sum B_{\lambda_{v}}$. Hence, by Hahn-Banach we need only see $A_{\lambda}^{\perp}=B_{\lambda}^{\perp}$ for each strongly dominant $\lambda$ to complete our proof.

Now (2.4) applies to each algebra:

$$
\begin{aligned}
L^{2}(\lambda) & =H^{2}(A, \lambda) \oplus \overline{H_{0}^{2}(A, \lambda)} \oplus(\operatorname{Re} A) \frac{1}{2} \\
& =H^{2}(B, \lambda) \oplus \overline{H_{0}^{2}(B, \lambda)} \oplus(\operatorname{Re} B)_{\frac{1}{2}},
\end{aligned}
$$

and since

$$
A \subset B, \quad H^{2}(A, \lambda) \subset H^{2}(B, \lambda), \quad \overline{H_{0}^{2}(A, \lambda)} \subset \overline{H_{0}^{2}(B, \lambda)} ;
$$

and $(\operatorname{Re} A)_{2}^{\frac{1}{2}} \subset(\operatorname{Re} B)_{\frac{1}{2}}$ as well since $(\operatorname{Re} A)_{\frac{1}{2}} \cdot \lambda=(\operatorname{Re} A)^{\perp} \cap L_{2}(\lambda) \cdot \lambda=(\operatorname{Re} B)^{\perp} \cap$ $L_{2}(\lambda) \cdot \lambda=(\operatorname{Re} B)_{2}^{\frac{1}{2}} \cdot \lambda$. So we have equality in each case, in particular

$$
H^{2}(A, \lambda)=H^{2}(B, \lambda) \text {. }
$$

We have $B^{\perp} \subset A^{\perp}$, so $B_{\lambda}^{\perp} \subset A_{\lambda}^{\perp}\left(\subset L_{1}(\lambda) \cdot \lambda\right)$. To see we have equality, let $h \in L^{\infty}(\lambda)$ be orthogonal to $B_{\lambda}^{\perp}$. Then $h$ is orthogonal to $\left(H_{0}^{2}(B, \lambda)+(\operatorname{Re} B)_{2}^{\perp}\right) \cdot \lambda \subset B_{\lambda}^{\perp}$, hence by (2.5) $h \in H^{2}(B, \lambda)$. But by (2.6) $h \in H^{2}(A, \lambda)$, and so by Theorem 2.9 we have $h$ in the weak* closure of $A$ in $L^{\infty}(\lambda)$; thus $h \perp A_{\lambda}^{\perp}$ and $A_{\lambda}^{\perp} \subset B_{\lambda}^{\perp}$, so $A_{\lambda}^{\perp}=B_{\lambda}^{\perp}$. As we saw this completes our proof.

The main part of this argument applies more generally.

CoRollary 2.12. Let $A \subset B$ be closed subalgebras of $C(X)$ and let $\lambda$ be a measure multiplicative on $B$ which is strongly dominant for the induced functional on $A$. Then $A_{\lambda}^{\perp}=B_{\lambda}^{\perp}$ iff $H^{2}(A, \lambda)=H^{2}(B, \lambda)$ (or equivalently by $(2.5)$, iff $(\operatorname{Re} A)_{2}^{\frac{1}{2}}=(\operatorname{Re} B)_{\frac{1}{2}}^{\frac{1}{2}}$ in the notation used there). 
Suppose the $H^{2}$ spaces coincide. We have $B_{\lambda}^{\perp} \subset A_{\lambda}^{\perp}$, and if $h \in L^{\infty}(\lambda)$ is orthogonal to $B_{\lambda}^{\perp}$ we obtain $h \in H^{2}(B, \lambda)$ as before. (This uses the multiplicativity of $\lambda$ on $B$; otherwise the decomposition in (2.5) need not be orthogonal.) Since then $h \in H^{2}(A, \lambda)$ we again conclude that $h \perp A_{\lambda}^{\perp}$ as in the previous proof. So $A_{\lambda}^{\perp}=B_{\lambda}^{\perp}$.

Conversely, if $A_{\lambda}^{\perp}=B_{\lambda}^{\perp}$ their intersections with $L^{2}(\lambda) \cdot \lambda$ coincide, so that $H_{0}^{2}(A, \lambda)$ $+(\operatorname{Re} A)_{2}^{\frac{1}{2}}=H_{0}^{2}(B, \lambda)+(\operatorname{Re} B)_{2}^{\frac{1}{2}}$, whence $H^{2}(A, \lambda)=H^{2}(B, \lambda)$ by $(2.5)$.

COROLlary 2.13. Suppose $A \subset B$ as before, and share the same set of multiplicative measures. Suppose moreover that on $A$ distinct multiplicative measures induce distinct functionals (so $M_{\phi}$ is a singleton for each $\phi \in \mathfrak{M}$, or representing measures are "unique"). Then $\sum A_{\lambda}^{\perp}=\sum B_{\lambda}^{\perp}$ in the notation of (2.3), and $A=B$ if the completely singular orthogonal measures for the two algebras coincide.

Each multiplicative measure $\lambda$ is of course strongly dominant for the corresponding homomorphism $\phi$ on $A$ or $B$, so that (2.3) holds. And as is well known $[\mathrm{L}, \mathrm{H} 1]$, uniqueness of the representing measure $\lambda$ for $\phi$ implies

$$
\begin{aligned}
L^{2}(\lambda) & =H^{2}(A, \lambda) \oplus \overline{H_{0}^{2}(A, \lambda)} \\
& =H^{2}(B, \lambda) \oplus \overline{H_{0}^{2}(B, \lambda)}
\end{aligned}
$$

so that $H^{2}(A, \lambda)=H^{2}(B, \lambda)$. So by $2.12, A_{\lambda}^{\perp}=B_{\lambda}^{\perp}$, yielding our first assertion; the second follows from 2.8 .

We should note a related consequence of the preceding.

COROLlaRY 2.14. Let $A$ be a closed subalgebra of $C(X)$ and suppose that in each nontrivial part we have some $\phi$ with a strongly dominant representing measure $\lambda$, with $\Lambda$ the collection of all such $\lambda$. Suppose there are no completely singular orthogonal measures. Then

$$
A=\left\{f \in C(X): f \in H^{2}(\lambda) \text { for all } \lambda \text { in } \Lambda\right\} .
$$

The right side is an algebra (by 2.9 , or directly) containing $A$, but all we need note is that if one of its elements $f$ is not in $A$ there is a $: \mathcal{i}$ in $A^{\perp}$, hence one in $A_{\lambda}^{\perp}$ for some $\lambda \in \Lambda$, not orthogonal to $f$. Since $f \in H^{2}(\lambda) \cap L^{\infty}(\lambda)=H^{\infty}(\lambda)$, by 2.9 $a_{n} \rightarrow f$ weak $^{*}$ for some sequence $\left\{a_{n}\right\}$ in $A$, so $\mu(f)=\lim \mu\left(a_{n}\right)=0$.

We should note that $H^{\infty}(\lambda)$ (or the set in 2.14) is always an algebra. For $f \in H^{\infty}(\lambda)$ implies $A f \subset H^{2}(\lambda)$, whence $f \cdot H^{2}(\lambda) \subset H^{2}(\lambda)$ since $f$ is bounded, so

$$
f H^{\infty} \subset H^{2} \cap L^{\infty}=H^{\infty} .
$$

3. Rational approximation in the plane. Let $K$ be a compact subset in the complex plane $C$, and let $R(K)$ denote the closure in $C(K)$ of the rational functions with poles off $K, A(K)$ the larger closed subalgebra of $C(K)$ of functions analytic on $K^{0}$, the interior of $K$. For each of these algebras evaluation at the elements of $K$ provides the elements of $\mathfrak{M}$ [Ar], and we may identify $\mathfrak{M}$ and $K$. And in each 
case $\partial K$, the topological boundary, provides the Šlov boundary of the algebra, which we shall always take as the $X$ of $\S 2$. It will be convenient to speak sometimes of $R(K)$ and $A(K)$ as subalgebras of $C(\partial K)$ (meaning of course the isomorphic algebras of restrictions), and in this section we shall always consider orthogonal measures carried only by $\partial K$. We shall consider $K$ as a subset of the Riemann sphere $S$ so that the point at infinity will play no special rôle, but we shall always want it to lie outside $K$.

Fundamental to our considerations will be a theorem of Walsh [Wa 1] which can be stated on $S$ as follows: if $U$ is a nonvoid connected open subset of $S$ and $K=S \backslash U$, then $R(K)$, as an algebra on $\partial K$, is $\operatorname{dirichlet}\left({ }^{4}\right)$. This implies [We 3] that each $z \in \partial K$ is a peak point for some $f$ in $R(K)$ (so, by definition, $\partial K$ is the minimal boundary [B 2] for $R(K)$ ). Thus if $K$ is a compact proper subset of $S$ whose boundary is just the union of the boundaries of the components of $S \backslash K$, then each $z$ in $\partial K$ is a peak point for $R(K)$, hence for $A(K)$, so $\partial K$ is the minimal boundary for $A(K)$. In all that follows we shall consider only $K$ for which $\partial K$ is the minimal boundary for $A(K)$, in particular those for which $S \backslash K$ has finitely many components.

The fact that $\partial K$ is the minimal boundary for $A(K)$ implies $\left(^{5}\right)[\mathrm{T}]$ that any continuous function $f$ on $\partial K$ has a continuous extension $\hat{f}$ to $K$ harmonic on $K^{0}$ (i.e., the dirichlet problem is solvable). This allows a simple definition of harmonic measures, and a brief introduction of these is perhaps in order. The map $f \rightarrow \hat{f}$ of $C^{R}(\partial K)$ into continuous (real-valued) harmonic functions is of course order preserving, so for $z \in K, f \rightarrow \hat{f}(z)$ is a nonnegative linear functional, 1 at 1 ; the corresponding (unique) probability measure $\lambda_{z}\left(=\delta_{z}\right.$ if $\left.z \in \partial K\right)$ on $\partial K$ is the harmonic measure for $z$, and as is easily seen, is carried (for $z \in K^{0}$ ) by the boundary of the component of $K^{0}$ containing $z$ (take $f \in C^{R}(\partial K)$ peaking on that part of $\partial K)$. Each $\lambda_{z}$ is a multiplicative measure on $A(K)$, since $f(z)=\int f d \lambda_{z}$ for $f \in A(K)$, $f$ being continuous and harmonic. Moreover $z \rightarrow \lambda_{z}$ is weak* continuous (as a map into $C^{R}(\partial K)^{*}$ ) since each $\hat{f} \in C(K)$.

Because of this continuity if $\mu$ is any measure carried by $K$ we can form the measure on $\partial K$ given by the weak* convergent integral

$$
\int \lambda_{z} \mu(d z)
$$

doing so is "sweeping the measure $\mu$ to $\partial K$ ", and we have the swept measure (3.1) and $\mu$ agreeing on $A(K)$, since $\int f(z) d \mu=\int \lambda_{z}(f) \mu(d z), f \in A(K)$. One use of sweeping is to see that

3.1. If we wish to show the real measures $\mu$ on $\partial K$ orthogonal to $R(K)$ have an at most $n$-dimensional span we need only prove this for some sequence $\left\{K_{v}\right\}$ of larger compacta which decrease to $K$.

$\left.{ }^{4}\right)$ I.e., $\operatorname{Re} R(K)$ is dense in $C^{R}(\partial K)$. A more direct "functional analytic" proof of Walsh's theorem is the content of the first three lemmas of [C].

$\left({ }^{5}\right)$ We shall require a minimal acquaintance with basic facts concerning harmonic functions, as covered for example in pp. 1-11 of [T]. 
For if $\lambda_{z}^{v}$ is the harmonic measure for $z$ relative to $K_{v}$ then $\mu_{v}=\int \lambda_{z}^{v} \mu(d z)$ is a real measure on $\partial K_{v}$ orthogonal to $R\left(K_{v}\right)$, and $\mu_{v} \rightarrow \mu$ weak* since any weak* cluster point of $\lambda_{z}^{v}$ will be a measure on $\partial K$ representing $z$ on rational functions, hence coinciding with $\delta_{z}$ (since $\partial K$ is assumed the minimal boundary of $A(K)$ ). So $\lambda_{z}^{v} \rightarrow \delta_{z}$ weak*, whence $\mu_{\nu} \rightarrow \mu$ weak* by dominated convergence. Thus if the span of the swept measures $\mu_{v}$ on $\partial K_{v}$ is at most $n$-dimensional for each $\nu$, the same is true of the span of the $\mu$.

Two other properties of harmonic measures will be useful. First $z \rightarrow \lambda_{z}$ is norm continuous on $K^{0}$. This is evident if $K$ is a closed disc $D$ (where harmonic measure is the Poisson kernel multiplying Lebesgue measure on the boundary), and if $D$ is a closed disc in $K^{0}$ then for $z$ in $D$ sweeping $\lambda_{z}^{D}$ out to $\partial K$ yields $\lambda_{z}: \lambda_{z}=\int \lambda_{w} \lambda_{z}^{D}(d w)$. So if $\left\|\lambda_{z}^{D}-\lambda_{z^{\prime}}^{D}\right\|<\varepsilon,\left\|\lambda_{z}-\lambda_{z^{\prime}}\right\|=\left\|\int \lambda_{w}\left(\lambda_{z}^{D}-\lambda_{z^{\prime}}^{D}\right)(d w)\right\|<\varepsilon$. The final fact we need can also be obtained by sweeping (several) $\lambda_{z}^{D}$ out to $\partial K$ : if $z_{1}, z_{2}$ lie in the same component of $K^{0}, \lambda_{z_{1}}$ and $\lambda_{z_{2}}$ are boundedly equivalent measures (i.e., with bounded Radon-Nikodym derivatives).

As indicated earlier, our attack on Mergelyan's rational approximation theorem using the results of $\S 2$ follows from an evaluation of the defect, in $C^{R}(\partial K)$, of $(\operatorname{Re} R(K))^{-}$, and of $(\operatorname{Re} A(K))^{-}$, or alternatively of the dimensions of the spans of the real orthogonal measures carried by $\partial K$. For convenience we shall call the defect of $(\operatorname{Re} R(K))^{-}$the rational defect, and use analytic defect when the algebra is $A(K)$. The determination of the rational defect when $S \backslash K$ has finitely many components will be one of the main results of this section, reproducing the AhernSarason result in Theorem 3.13: the defect is the sum of the connectivities of the components of $K^{0}$. However we feel the main interest in our proof, which is long and involved, lies more in further applications of the methods used; consequently we have interposed many remarks only needed after 3.13 , and the reader might be well advised to eliminate certain portions of the text on first reading. Thus one can arrive at the Ahern-Sarason result by reading only Lemmas 3.2, 3.4, 3.5, the first part of Lemma 3.6, and then 3.9 through 3.13 (omitting all parenthetical remarks as well).

When $S \backslash K$ has $n+1$ components $H_{0}, \ldots, H_{n}$ a second theorem of Walsh [Wa 2] shows the rational defect is at most $n$ (the first cited theorem corresponds to the case $n=0$ ), so that strongly dominant representing measures exist for either algebra and each $z \in K(2.3 \mathrm{c})$. When the $H_{i}$ have disjoint boundaries $F_{i}$ the characteristic functions $\chi_{F_{1}}, \ldots, \chi_{F_{n}}$ are independent elements of $C^{R}(\partial K)$ not approximable by $\left({ }^{6}\right)$

${ }^{(6)}$ If $f \in A(K)$ and $\operatorname{Re} f$ approximates $\chi_{F_{1}}$ well, then $f(K)$ lies in two disjoint vertical strips about $\operatorname{Re} z=0, \operatorname{Re} z=1$, and applying to $f$ an analytic function $=0$ on the left strip and $=1$ on the right we obtain an element $g$ in $A(K)$ which has $g \mid \partial K=\chi_{F_{1}}$. But then $g(K)=\{0,1\}$, and $g$ provides a separation $K=A \cup B$ of $K$ where $g(A)=1, g(B)=0$, and $F_{1} \subset A$. It is easy to see $\partial A=F_{1}=\partial H_{1}$, and since each $z \in \partial H_{1}$ has a neighborhood lying wholly within $A \cup H_{1}$ we can extend $g$ to $K_{1}=K \cup H_{1}$ by setting $g=1$ on $H_{1}$ to obtain $g \in A\left(K_{1}\right)$ which is $\equiv 0$ on $\partial K_{1}=\bigcup_{j \neq 1} F_{j}$, and yet is nonzero. (We can. also see the analytic defect is at least $n$ by exhibiting $n$ real independent orthogonal measures, as in 3.13 below.) 
$\operatorname{Re} A(K)$. Thus the analytic defect is at least $n$, whence both defects coincide, $(\operatorname{Re} R(K))^{\perp}=(\operatorname{Re} A(K))^{\perp}$, and 2.11 applies once we see (in 3.6) there are no completely singular orthogonal measures.

Actually the first cited theorem of Walsh will suffice for our purposes, because of the following lemma which allows us to combine certain dirichlet algebras. Besides later utility it also has applicability to subalgebras of $A(K)$ other than $R(K)$.

LEMmA 3.2. Suppose $K=K_{0} \cap K_{1}$, and we are given closed subalgebras $A_{i}$ of $C\left(K_{i}\right)$ with Silov boundaries $\partial_{i}=\partial K_{i}, i=0,1$ for which each $f$ in $A_{i}$ is analyti: on $K_{i} \mid \partial_{i}$. Suppose $A_{0}$ is dirichlet on $\partial_{0}$, while $\left(\operatorname{Re} A_{1}\right)^{\perp}\left(\right.$ on $\left.\partial_{1}\right)$ is $n$-dimensional, and $\partial_{1}$ lies on one component $U$ of the interior of $K_{0}$. Then if $A$ is the closed subalgebra of $C(K)$ generated by $A_{0}$ and $A_{1}, \partial=\partial_{0} \cup \partial_{1}$ is the Silov boundary of $A$ and

(i) $(\operatorname{Re} A)^{\perp}\left(\right.$ on $\partial$ of course) is at most $(n+1)$-dimensional $\left({ }^{7}\right)$.

(ii) For $\mu \in(\operatorname{Re} A)^{\perp}, \mu_{\partial_{0}}$ is absolutely continuous with respect to $\lambda_{z}^{U}$, for any $z \in U$.

[The same sort of argument works when $\partial_{i}$ is a larger subset of $K_{i}$. So, for example, we could let $K_{0}=K_{1}=S$, and let $\partial_{0}$ and $\partial_{1}$ be disjoint replicas of Wermer's arc for which $A_{i}=\left\{f \in C(S): f\right.$ analytic on $\left.S \backslash \partial_{i}\right\}$ is dirichlet on $\partial_{i}$ [We 1]. Then the algebra $A$ has an at most one-dimensional space of real orthogonal measures on $\partial=\partial_{0} \cup \partial_{1}$; replacing $A_{1}$ by this algebra and introducing another arc $\partial_{2}$ disjoint from $\partial_{0} \cup \partial_{1}$ the argument applies again. So if $\partial_{0}, \ldots, \partial_{n}$ are a pairwise disjoint collection of such arcs the algebra we finally generate, and so that larger algebra of $f$ in $C(S)$ analytic off $\partial=\bigcup_{i=0}^{n} \partial_{i}$, has an at most $n$-dimensional space of real orthogonal measures on its Silov boundary (and one can easily see these are all boundedly absolutely continuous with respect to the harmonic measure for $S \backslash \partial$, as in 3.13 below). Indeed the dimension is precisely $n$, as one can see as in footnote 6 . Finally, suppose $\partial_{0}, \partial_{1}, \ldots, \partial_{n}$ are all interior to the unit disc $D$; then the algebra $A$ of $f \in C(D)$ analytic on $D^{0} \backslash \bigcup_{i=0}^{n} \partial_{i}$ has an $(n+1)$-dimensional space of real orthogonal measures.]

To begin the proof of 3.2 , let $\nu_{1}, \ldots, \nu_{n}$ be the real measures on $\partial_{1}$ spanning $\left(\operatorname{Re} A_{1}\right)^{\perp}$, and let $\mu$ be a real measure on $\partial=\partial_{0} \cup \partial_{1}$ orthogonal to $A$. Let $\mu_{i}=\mu_{\partial_{i}}$. Since $\partial K_{i}=\partial_{i}, \partial_{i}$ carries the harmonic measure $\lambda_{z}^{i}$ of $z \in K_{i}$ relative to $K_{i}$. Sweeping $\mu_{1}$ to $\partial_{0}$ we have $\mu_{0}+\int \lambda_{z}^{0} \mu_{1}(d z)$ a real measure on $\partial_{0}$ orthogonal to $A_{0}$ since $f \in A_{0} \subset A$ implies $\mu_{0}(f)+\int \lambda_{z}^{0}(f) \mu_{1}(d z)=\mu_{0}(f)+\int f(z) \mu_{1}(d z)=\left(\mu_{0}+\mu_{1}\right)(f)=0$. Since $A_{0}$ is dirichlet on $\partial_{0}$,

$$
\mu_{0}=-\int \lambda_{z}^{0} \mu_{1}(d z)
$$

Similarly $\mu_{1}+\int \lambda_{w}^{1} \mu_{0}(d w)$ is a real measure on $\partial_{1}$ orthogonal to $A_{1}$, whence we have real $c_{i}$ with

$$
\mu_{1}+\int \lambda_{w}^{1} \mu_{0}(d w)=-\sum_{i=1}^{n} c_{i} \nu_{i}
$$

$\left.{ }^{7}\right)$ Since $(\operatorname{Re} A)^{\perp}$ is spanned by real measures it is sufficient to show its real elements form a real space of dimension $\leqq n+1$. 
Since $\partial_{1} \subset U, \lambda_{z}^{0}=\lambda_{z}^{U}$ for $z \in \partial_{1}$ and thus (3.2) implies (ii), all measures $\lambda_{z}^{0}, z \in U$, being equivalent. If we let $X$ denote the closed carrier of any of the equivalent measures $\lambda_{z}^{0}, z \in \partial_{1}$, then $X \subset \partial U \subset \partial K_{0} \subset \partial_{0}$ carries $\mu_{0}$ by (3.2).

Combining (3.2) and (3.3)

$$
\mu_{0}=\iint \lambda_{z}^{0} \lambda_{w}^{1}(d z) \mu^{0}(d w)+\sum c_{i} \int \lambda_{z}^{0} \nu_{i}(d z)
$$

or, setting $\eta_{w}=\int \lambda_{z}^{0} \lambda_{w}^{1}(d z)$, a probability measure on $X$, and $\sigma_{i}=\int \lambda_{z}^{0} \nu_{i}(d z)$, again a measure on $X$,

$$
\mu_{0}=\int \eta_{w} \mu_{0}(d w)+\sum_{i=1}^{n} c_{i} \sigma_{i}
$$

Now set $T f(w)=\eta_{w}(f), f \in C^{R}(X)$. This defines a nonnegative bounded linear operator on $C^{R}(X)$ for which (3.5) says

$$
\left(I-T^{*}\right) \mu_{0}=\sum_{i=1}^{n} c_{i} \sigma_{i}
$$

As we observed $w \rightarrow \lambda_{w}^{1}$ is strongly continuous on $K_{1}^{0}$, and since $\partial_{0}$ is disjoint from $U \supset \partial_{1} \supset \partial K_{1}$ while $\partial_{0} \subset K \subset K_{1}, \partial_{0} \subset K_{1}^{0}$ so that $w \rightarrow \lambda_{w}^{1}$ in particular is strongly continuous on $\partial_{0}$. This implies $T$ takes the unit ball of $C^{R}(X)$ into an equicontinuous set:

$$
\left|f(w)-f\left(w^{\prime}\right)\right|=\left|\int \lambda_{z}^{0}(f)\left(\lambda_{w}^{1}-\lambda_{w^{\prime}}^{1}\right)(d z)\right| \leqq\|f\|\left\|\lambda_{w}^{1}-\lambda_{w^{\prime}}^{1}\right\| .
$$

Hence $T$ is a compact operator.

Since $T 1=1,1$ is an eigenvalue of $T$; in fact the nullity of $(I-T)$, and of $(I-T)^{2}$, is spanned by the constant function 1 . To see this note that $\eta_{w}$ has carrier precisely $X$ so that if $f(w)=\eta_{w}(f)$ for all $w$ in $X$ for some $f \in C^{R}(X)$, selecting $w$ so as to maximize $f(w)$ shows $f$ assumes this value on all of $X$. Again if $(I-T)^{2} f=0,(I-T) f$ is a constant, so $f(w)-\eta_{w}(f)=c$. If $c>0$, (respectively $c<0$ ) selecting $w$ so that $f(w)$ is a minimum (respectively maximum) leads to a contradiction, so we conclude $c=0$ and have $f$ a constant as before.

So 1 is a simple eigenvalue of $T$, and so, by the Riesz theory of compact operators [RN], of $T^{*}$. Thus we have a one-dimensional nullity for $I-T^{*}$ and for $\left(I-T^{*}\right)^{2}$ $=(I-T)^{2 *}$, so that $\left(I-T^{*}\right)$ is invertible on the range of $I-T^{*}$, and the space of measures on $X$ is the direct sum of the nullity and range [RN].

If $\left(I-T^{*}\right) \tau_{0}=0, \tau_{0} \neq 0$ then since the space of measures on $X$ is the direct sum of nullity and range, we have a real $c_{0}$ for which $\mu_{0}-c_{0} \tau_{0}$ is in the range of $I-T^{*}$, and similarly real $k_{\mathfrak{i}}$ for which $\sigma_{\mathfrak{i}}-k_{\mathfrak{i}} \tau_{0}$ is in that range. By (3.6)

$$
\begin{aligned}
\left(I-T^{*}\right) \mu_{0} & =\left(I-T^{*}\right)\left(\mu_{0}-c_{0} \tau_{0}\right)=\sum c_{i} \sigma_{i} \\
& =\sum c_{i}\left(\sigma_{i}-k_{i} \tau_{0}\right)+\left(\sum c_{i} k_{i}\right) \tau_{0}
\end{aligned}
$$


and since all but the last term lie in the range of $I-T^{*}$ we conclude $\sum c_{i} k_{i}=0$, in view of our direct sum. Applying the inverse $S$ of the restriction of $I-T^{*}$ to its range we have

and so by (3.4)

$$
\mu_{0}-c_{0} \tau_{0}=\sum_{i=1}^{n} c_{i} \tau_{i}, \quad \tau_{i}=S\left(\sigma_{i}-k_{i} \tau_{0}\right)
$$

$$
\begin{aligned}
\mu_{1} & =-\int \lambda_{w}^{1} \mu_{0}(d w)-\sum c_{i} \nu_{i} \\
& =-\sum_{i=0}^{n} c_{i} \int \lambda_{w}^{1} \tau_{i}(d w)-\sum_{i=1}^{n} c_{i} \nu_{i}
\end{aligned}
$$

so the span of $\tau_{0}-\int \lambda_{w}^{1} \tau_{0}(d w), \tau_{i}-\int \lambda_{w}^{1} \tau_{i}(d w)-v_{i}, i=1, \ldots, n$, contains $(\operatorname{Re} A)^{\perp}$.

[REMARK 3.3. One further consequence of this proof should be noted. We have seen $T^{*}$ has a unique fixed point $\tau_{0}$. On the other hand $T^{*}$ carries the set of probability measures on $X$ into itself, so that some probability measure is a fixed point (most simply by the Markov-Kakutani theorem [DS]). Thus we can take $\tau_{0} \geqq 0$, and the first of the above real orthogonal measures, $=\tau_{0}$ on $\partial_{0}$ and $-\int \lambda_{w}^{1} \tau_{0}(d w)$ on $\partial_{1}$, is $\geqq 0$ on $\partial_{0}$ and $\leqq 0$ on $\partial_{1}$. This leads to some similarity between the situation for certain $R(K)$ and the familiar one when $K$ is an annulus, as we shall note later (cf. 3.13).]

3.4. In order to apply Lemma 3.2 to $R(K)$ we make the following observations which will reduce our considerations to connected $K$. Let $F$ be a component of $K$. Then

(a) the closure of $R(K) \mid F$ in $C(F)$ is $R(F)$ : for each component $V$ of $S \backslash F$ meets one of $S \backslash K$, so $R(K) \mid F$ contains the function $1 /(z-v)$ for some $v \in V$. But in order to generate $R(F)$ it is only necessary to use a pole at one element of each component of $S \backslash F$, so $R(K) \mid F$ is dense in $R(F)$ as asserted. As a consequence

(b) $R(F)^{\perp}$ consists of the measures in $R(K)^{\perp}$ carried by $F$, and $(\operatorname{Re} R(F))^{\perp}$ consists of those in $(\operatorname{Re} R(K))^{\perp}$ carried by $F$.

The standard fact that we may apply a function analytic near the spectrum of an element $f$ of $R(K)$ to $f$ and obtain an element of $R(K)$ yields for $f(z) \equiv z$ (which has spectrum $=K)$, the fact that we have a sequence $\left\{f_{n}\right\}$ of idempotents in $R(K)$ which tend pointwise to $\chi_{F}$. This shows the first part of

(c) $z \in F$ implies $M_{z}$ is carried by $F$, and coincides with the set of measures on $\partial F$ representing $z$ on $R(F)$ (i.e., $M_{z}(R(K))=M_{z}(R(F))$ ).

(The second assertion follows from the first and (a)). Another consequence of the existence of $\left\{f_{n}\right\}$ is that no set of antisymmetry [G1 1] meets $F$ and its complement; since $F$ is any component of $K$, and any extreme element of the unit ball of $R(K)^{\perp}$ is carried by a set of antisymmetry [Gl 1], we have

(d) any extreme point of the unit ball of $R(K)^{\perp}$ is carried by some component of $K$. And

(e) any extreme point $\mu$ of the unit ball of $(\operatorname{Re} R(K))^{\perp}$ is carried by some component $F$ of $K$, and so lies in $(\operatorname{Re} R(F))^{\perp}$. 
Indeed $\mu$ is carried by a set of antisymmetry by just the argument of [Gl 1] since for any $f \in R(K)$ real on the carrier of $\mu$ we clearly have $f \mu \in(\operatorname{Re} R(K))^{\perp}$. The last assertion follows from (b).

Now suppose we knew that for every connected $K$ for which $S \backslash K$ has finitely many components the dimension of $(\operatorname{Re} R(K))^{\perp}$ is one less than the number of components of $\partial K$, and we are given a disconnected $K$. As is easily seen $\partial F$ is connected for all components $F$ of $K$ except for a finite collection, $F_{1}, \ldots, F_{k}$ say, and for those with $\partial F$ connected we can apply the known result (since $F$ is connected and $S \backslash F$ has finitely many components) to conclude that $(\operatorname{Re} R(F))^{\perp}=0$. Thus the $\mu$ in (e) are each carried by one $F_{i}$, and we see that $(\operatorname{Re} R(K))^{\perp}=\sum_{i=1}^{k}\left(\operatorname{Re} R\left(F_{i}\right)\right)^{\perp}$, and so has a (finite) dimension equal to $\sum_{i=1}^{k} n_{i}$ if $\partial F_{i}$ has $n_{i}+1$ components.

Now to see that (for $K$ connected) $(\operatorname{Re} R(K))^{\perp}$ has dimension $n$ if $\partial K$ has $n+1$ components we need only prove $R(K)$ is dirichlet on $\partial K$ when $\partial K$ is connected. This reduction to the case $n=0$ is obtained from 3.2 by induction, as follows.

Since $K$ is connected each component $V$ (of the finitely many) of $S \backslash K$ is simply connected and has a connected boundary [N], and thus each component of $\partial K$ is the boundary of a union of such $V$. Let $B_{0}, \ldots, B_{n}$ denote the components of $\partial K$, $U_{0}, \ldots, U_{n}$ the corresponding unions of $V$ 's with $\partial U_{i}=B_{i}$. If any $B_{i}$ does not separate the remaining components of $\partial K$ (i.e., all others lie in one component of $S \backslash B_{i}$ ) we can apply 3.2 to $K_{0}=S \backslash U_{i}, K_{1}=S \backslash \bigcup_{j \neq i} U_{j}$ (so $K=K_{0} \cap K_{1}$ ) and $R\left(K_{0}\right)$, $R\left(K_{1}\right)$ since $R\left(K_{0}\right)$ is dirichlet on $\partial K_{0}=B_{i}$ and $\left(\operatorname{Re} R\left(K_{1}\right)\right)^{\perp}$ has dimension $n-1$ by our induction hypothesis; and so $(\operatorname{Re} R(K))^{\perp}$ has dimension $\leqq n$ (and $\geqq n$ by footnote 6).

But some $B_{i}$ does not separate the others by a simple argument. If for example $B_{i}$ separates so that, say, $B_{i+1}, B_{i+2}, \ldots, B_{n}$ lie in one component, with $B_{0}, \ldots, B_{i-1}$ in others then $B_{i}, B_{i+1}, \ldots, B_{n}$ lie in one component of $S \backslash B_{i-1}$ : for $B_{i-1}$ lies in one (simply connected) component $V$ of $S \backslash B_{i}$ which does not meet $B_{i+1}, \ldots, B_{n}$, or $B_{i}$, so that the connected complement $V^{\prime}$ contains $B_{i}, B_{i+1}, \ldots, B_{n}$ and must lie in one component of $S \backslash B_{i-1}$. So another choice of the separating $B_{i}$ leads to a larger number of $B_{j}$ in one component, and we can continue until we arrive at a nonseparating one.

In summary then

(f) once we see $R(K)$ is dirichlet on $\partial K$ when $K$ and $\partial K$ are connected (and $S \backslash K$ has finitely many components, of course) then whenever $S \backslash K$ has finitely many components if $F_{1}, \ldots, F_{k}$ are the components of $K$ with disconnected boundaries, while $\partial F_{i}$ has $n_{i}+1$ components, $(\operatorname{Re} R(K))^{\perp}$ has dimension $\sum_{i=1}^{k} n_{i}$.

Our discussion also shows how Walsh's earlier theorem [Wa 1] (on sets $K$ with $S \backslash K$ connected, cited at the beginning of this section) implies directly that $(\operatorname{Re} R(K))^{\perp}$ is finite dimensional (with $K$ as above). Indeed we need only see this when $K$ is connected, and then components $V$ of $S \backslash K$ are simply connected; shrinking every $V$ slightly to a smaller simply connected $\tilde{V}$ we can obtain a larger $K, \tilde{K}$, for which the components of $S \backslash \tilde{K}$ have disjoint closures, while each $R(S \backslash \tilde{V})$ is dirichlet on 
$\partial \tilde{V}=\partial(S \backslash \tilde{V})$ by Walsh's first result. As in the above argument we can then use induction and Lemma 3.2 to see $(\operatorname{Re} R(\tilde{K}))^{\perp}$ is finite dimensional, and 3.1 then yields the same fact for $K$.

[(g) One final remark should be made on the special case in which $K$ is connected and $\partial K$ has components $B_{0}, \ldots, B_{n}$ none of which separates the remainder: then one has a basis $\tau_{1}, \ldots, \tau_{n}$ of $(\operatorname{Re} R(K))^{\perp}$ with $\tau_{i} \geqq 0$ on $B_{i}$, $\leqq 0$ on $\bigcup_{j \neq i} B_{j}$ (and nonvanishing on each such $B$,). Indeed using $B_{i}$ as $\partial_{0}$ in Remark 3.3 we obtain a $\tau_{i} \geqq 0$ on $B_{i}$, $\leqq 0$ elsewhere. To see the measures $\tau_{1}, \ldots, \tau_{n}$ we obtain for $i=1, \ldots, n$ are independent we note that since $\tau_{i} \perp 1$,

$$
\sum_{j=1}^{n} \tau_{i}\left(B_{j}\right)=-\tau_{i}\left(B_{0}\right) \geqq 0,
$$

and that our result follows if we have strict inequality for every $i$ : for then the matrix $\left(a_{i j}\right), a_{i j}=\tau_{i}\left(B_{j}\right), i, j=1, \ldots, n$, has $a_{i i}>\sum_{j \neq i}\left|a_{i j}\right|\left(\right.$ i.e., $\left.\tau_{i}\left(B_{i}\right)>\sum_{j \neq i, 0}-\tau_{i}(B),\right)$, so that $\left(a_{i j}\right)$ is nonsingular by the familiar Gerschgorin criterion [MM].

Now if $\tau_{i}\left(B_{0}\right)=0$ we have $\lambda_{z}^{1}\left(B_{0}\right)=0$ for some $z$ in $B_{i}$ since off $B_{i}, \tau_{i}=-\int_{B_{i}} \lambda_{z}^{1} \tau_{i}(d z)$ as in 3.3 (where $\lambda_{z}^{1}$ is harmonic measure for $z$ relative to $K_{1}$ as above, in the argument establishing (f)). But then $\lambda_{z}^{1}\left(B_{0}\right)=0$ for all $z$ in the component $U$ of $K_{1}^{0}$ containing $B_{i}$. If we let $U_{j}$ again be the union of the components of $S \backslash K$ with boundary $\subset B_{j}$ then one component $W_{j}$ of $S \backslash B_{j}$, indeed of $S \backslash U_{j}^{-}$, contains the remaining $B_{k}$ by hypothesis, and by [N, p. 101, 8.2] $W_{j} \cap W_{k}$ is the component of $\left(S \backslash U_{j}^{-}\right) \backslash U_{k}^{-}$(hence of $S \backslash\left(B_{j} \cup B_{k}\right)$ ) containing the remaining $B_{l}$, since $U_{k}^{-} \subset S \backslash U_{j}^{-}$, the $U_{i}^{-}$being pairwise disjoint. And clearly $\partial\left(W_{j} \cap W_{k}\right)=\partial W_{j} \cup \partial W_{k}$. Applying the result several times we see $\bigcap_{j \neq i} W_{j}$ is the component $U$ of $K_{i}^{0}=S \mid\left(\bigcup_{j \neq i} U_{j}^{-}\right)$ containing $B_{i}$, and $\partial U=\bigcup_{j \neq i} \partial W_{j}$. Thus $\varnothing \neq \partial U \cap \partial W_{0} \subset \partial U \cap B_{0}$. But $\lambda_{z}^{1}\left(B_{0}\right)=0$ for $z$ in $U$ and therefore in $\partial U \cap B_{0}$ since $\hat{\chi}: z \rightarrow \lambda_{z}^{1}\left(B_{0}\right)$ is the continuous harmonic function on $K_{1}$ corresponding to the element $\chi_{B_{0}}$ of $C\left(\partial K_{1}\right)$; but for the same reason $\hat{\chi}(z)=1$ on $B_{0}$, our contradiction, and we conclude that $\tau_{i}\left(B_{0}\right) \neq 0, i \neq 0$. By the same argument $\tau_{i}\left(B_{j}\right) \neq 0$ if $i \neq j$.]

3.5. In order to apply the results of $\S 2$ to rational approximation we shall need three lemmas. The first reproduces a step in [Gl W] to show we have no completely singular measures in $R(K)^{\perp}$ when $S \backslash K$ has finitely many components. The result depends on the well-known result of Bishop [B 2] that $R(K)=C(K)$, if (and only if) the minimal boundary for $R(K)$ fills out all of $K$ except for a set of plane measure zero (so in particular if $\partial K$ is the minimal boundary for $R(K)$, hence for $R(\partial K)$, $R(\partial K)=C(\partial K))$.

LEMMA 3.6. If $R(\partial K)=C(\partial K)$, and we have a dominant representing measure on $R(K)$ for each $z \in K$, then there are no completely singular measures orthogonal to $R(K)$.

Further, suppose we also have $\partial K$ the minimal boundary for $R(K)$ (so that $R(\partial K)$ 
$=C(\partial K)$ and each nontrivial Gleason part for $R(K)$ is a union of components $\left(^{8}\right)$ of $\left.K^{0}\right)$. If $Q$ is any collection of nontrivial parts, with (open) union $U$, and we select one dominant representing measure $\lambda$ for one point in each part not in $Q$ to form the collection $\Lambda$, and set $K_{Q}=K \mid U$ then

$$
R\left(K_{Q}\right)^{\perp}=\sum_{\Lambda} R(K)_{\lambda}^{\perp}
$$

(where the sum denotes all norm convergent series as before). Finally, if in addition harmonic measures are dominant, $R\left(K_{Q}\right)^{\perp}=R\left(K_{Q}^{0-}\right)^{\perp}$.

Note that $\partial K_{Q}=\partial K$, so that in the second part of $3.6 \partial K_{Q}$ is the minimal boundary of the algebra $R\left(K_{Q}\right) \supset R(K)$. (We can naturally view $R(K)$ as a subalgebra of $R\left(K_{Q}\right)$ by identifying $R(K)$ and $R(K) \mid K_{Q}$.) To begin the proof note that if $\sigma$ is a completely singular element of $R(K)^{\perp}$, and $\lambda$ is any dominant representing measure for $z_{0} \in K^{0}$, then

$$
\sigma /\left(z-z_{0}\right)-\sigma\left(1 /\left(z-z_{0}\right)\right) \cdot \lambda
$$

is orthogonal to $C+\left(z-z_{0}\right) \cdot R(K)=R(K)$ so by Theorem 2.6 its singular part with respect to $\lambda, \sigma /\left(z-z_{0}\right)$, is also. This same argument applies to $\sigma^{1}=\sigma /\left(z-z_{0}\right)$ to show

$$
1 /\left(z-z_{0}\right) \cdot 1 /\left(z-z_{1}\right) \cdot \sigma
$$

is orthogonal to $R(K), z_{1} \in K^{0}$, and continuing we see $\sigma$ is orthogonal to the subalgebra of $C(\partial K)$ generated by $R(K)$ and all functions $z \rightarrow 1 /\left(z-z_{0}\right), z_{0} \in K^{0}$, hence to $R(\partial K)=C(\partial K)$. So $\sigma=0$.

Similarly if we now take $z_{0} \in U$ with dominant representing measure $\lambda_{0}$, then $\lambda \in \Lambda$ and $\lambda_{0}$ are mutually singular and if $\mu \in R(K)_{\lambda}^{\perp}$, we have

$$
\mu /\left(z-z_{0}\right)-\mu\left(1 /\left(z-z_{0}\right)\right) \lambda_{0}
$$

orthogonal to $C+\left(z-z_{0}\right) R(K)=R(K)$, so that $\mu /\left(z-z_{0}\right) \perp R(K)$; as before we can conclude that $\mu \perp R\left(K_{Q}\right)$.

Now since we may view $R(K)$ as a subalgebra of $R\left(K_{Q}\right)$ in $C(\partial K)$, each measure multiplicative on $R\left(K_{Q}\right)$ is multiplicative on $R(K)$, and of course represents a point $z \in K_{Q}$. On the other hand for $z \in K_{Q}^{0}$ if $\lambda^{1} \in M_{z}$ for $R(K)$, and so is in $L^{1}(\lambda)$ for some $\lambda$ in $\Lambda$, then $\mu=\lambda_{z}-\lambda^{1}$ is orthogonal to $R(K)$, and thus orthogonal to $R\left(K_{Q}\right)$ as we have just seen. Since $\lambda_{z}$ is multiplicative on $R\left(K_{Q}\right)$, the same is true of $\lambda^{1}$ : so $z \in K_{Q}^{0}$ has the same set of representing measures on $\partial K_{Q}=\partial K$ for $R(K)$ and $R\left(K_{Q}\right)$. The same is of course the case for $z \in \partial K_{Q}$, a peak point, so that the representing measures for $R\left(K_{Q}\right)$ are just those for $R(K)$ which represent points in $K_{Q}$.

We have two consequences: dominant representing measures are available for $R\left(K_{Q}\right)$ (so that $R\left(K_{Q}\right)^{\perp}$ has no completely singular elements by the argument

${ }^{8}{ }^{8}$ Each component lies in a part $P$, and since boundary points form singleton parts, $P \subset K^{0}$, and so is a union of components of $K^{0}$. 
applied to $R(K)$ ) and the nontrivial parts for $R\left(K_{Q}\right)$ are simply the nontrivial parts for $R(K)$ contained in $K_{Q}$, i.e., those not in $Q$. Thus $R\left(K_{Q}\right)^{\perp}=\Sigma_{\Lambda} R\left(K_{Q}\right)_{\lambda}^{\perp}$ by 2.8 .

But since $R(K) \subset R\left(K_{Q}\right)$ we have $R\left(K_{Q}\right)^{\perp} \subset R(K)^{\perp}$, and so $R\left(K_{Q}\right)_{\lambda}^{\perp} \subset R(K)_{\lambda}^{\perp}$; on the other hand we have seen $R(K)_{\lambda}^{\perp} \subset R\left(K_{Q}\right)^{\perp}$, hence $\subset R\left(K_{Q}\right)_{\lambda}^{\perp}$, for $\lambda \in \Lambda$, so $R\left(K_{Q}\right)_{\lambda}^{\perp}=R(K)_{\lambda}^{\perp}$, and this implies (3.7).

For the final fact, that $R\left(K_{Q}\right)^{\perp}=R\left(K_{Q}^{0-}\right)^{\perp}$ when harmonic measures are dominant, note first that then each measure in the first set is carried by $\partial K_{Q}^{0}$ since each $\lambda$ in $\Lambda$ is. Since each component of $S \backslash K_{Q}^{0-}$ must meet a component of $S \backslash K_{Q}$ ( $\partial K_{Q}$ being nowhere dense) and since only one pole is needed in each complementary component to generate $R\left(K_{Q}^{0-}\right), R\left(K_{Q}\right) \mid K_{Q}^{0-}$ is dense in $R\left(K_{Q}^{0-}\right)$, so that $R\left(K_{Q}\right)^{\perp}$ $\subset R\left(K_{Q}^{0-}\right)^{\perp}$. The reverse inclusion is trivial, and our proof complete.

The crucial property of $R(K)$, that $R(K)=C+\left(z-z_{0}\right) R(K)$ for $z_{0} \in K^{0}$, is shared by $A(K)$, so by the same arguments we obtain

COROLlaRY 3.7. If $K$ satisfies the hypotheses of 3.6, while $U, Q$ and $\Lambda$ are defined in terms of the parts for $A(K)$, then $\Sigma_{\Lambda} A(K)_{\lambda}^{\perp}=B^{\perp}$, where $B$ is the subalgebra of $C\left(K_{Q}\right)$ generated by $A(K)$ and all functions $z \rightarrow 1 /\left(z-z_{0}\right), z_{0} \in U$.

Note that since fewer measures on $\partial K$ represent $z \in K$ on $A(K)$ than on $R(K)$, we have dominant representing measures for $A(K)$ by hypothesis.

Lemma 3.6 will (later) provide some easy extensions of Mergelyan's results, but now we should note

COROLlaRY 3.8. Under the hypotheses of 3.6 (respectively 3.7) $\sum_{\Lambda} R(K)_{\lambda}^{\frac{1}{1}}$ (respectively $\sum_{\Lambda} A(K)_{\lambda}^{\perp}$ ) is weak* closed.

In particular each summand $R(K)_{\lambda}^{\perp}$ is a weak* closed space of measures.

Finally the argument in $\mathbf{3 . 6}$ showing there are no completely singular orthogonal measures can be used in other instances when the crucial property that $A=C$ $+\left(z-z_{0}\right) A$ holds for sufficiently many $z_{0}$ : for example, the algebra $A$ mentioned after the statement of 3.2, consisting of all functions in $C(D)$ analytic on $U=$ $D^{0} \backslash \bigcup_{i=0}^{n} \partial_{i}$ has this property for all $z_{0}$ in $U$; since each point $z$ of $\partial U$ is a peak point for $R(\partial U)-z$ in $\partial_{i}$ being a peak point for Wermer's algebra $A_{i}$-the cited theorem of Bishop [B 2] applies to show $R(\partial U)=C(\partial U)$, and thus $A^{\perp}$ contains no completely singular measures. The same applies to its subalgebra $B$ generated by all functions analytic on $D \backslash \bigcup_{i=0}^{n} \partial_{i}$, i.e., each analytic on $(1+\varepsilon) D \backslash \bigcup_{i=0}^{n} \partial_{i}$ for some $\varepsilon>0$. Both algebras have $\mathfrak{M}=D$, as one sees from [Ar], and since $(\operatorname{Re} B)^{\perp}$ and $(\operatorname{Re} A)^{\perp}$ both have dimension $n+1$ (by the argument given after the statement of 3.2) $A=B$ by 2.11.

Our second lemma is an assertion about harmonic measures which must be well known, although we have not been able to find it in the literature; in the simplest case it essentially says that if we punch a hole in a domain $V$ to obtain a new one $U$ the old harmonic measure (for $z \in U$ ) is absolutely continuous with respect to the new one away from the boundary of the hole. I am indebted to Maynard G. Arsove for suggesting its proof. 
We shall use the following notation. Since $K^{0}$ has countably many components, we select one point $z_{n}$ in the $n$th component, and let $\lambda_{n}$ be its harmonic measure. Then $\lambda^{*}$ will denote the measure $\sum 2^{-n} \lambda_{n}$.

LEMMA 3.9. Let $K=K_{0} \cap K_{1}$ where each compact $K_{i}$ has its boundary as the minimal boundary for $R\left(K_{i}\right)$ and $\partial K=\partial K_{0} \cup \partial K_{1}$. Let $\lambda_{z}^{i}$ be the harmonic measure for $z \in K_{i}$ relative to $K_{i}$, and $\lambda_{z}$ that for $z \in K$ relative to $K$.

Let $U$ be a component of $K^{0}$, and $U_{i}$ the component of $K_{i}^{0}$ containing $U$ (so $\left.U=U_{0} \cap U_{1}\right)$. Then for $z \in U, \lambda_{z}^{0}$ (respectively $\lambda_{z}^{1}$ ) is absolutely continuous with respect to $\lambda^{*}$ on $\partial U_{0} \mid \partial K_{1}$ (respectively $\left.\partial U_{1} \mid \partial K_{0}\right)$.

In particular, if $\left(\partial U_{0} \mid \partial K_{1}\right) \cup\left(\partial U_{1} \mid \partial K_{0}\right) \subset \partial U, \lambda_{z}^{0}+\lambda_{z}^{1}$ is absolutely continuous with respect to $\lambda_{z}$ on $\partial U \backslash\left(\partial K_{0} \cap \partial K_{1}\right)\left(\subset\left(\partial U_{0} \mid \partial K_{1}\right) \cup\left(\partial U_{1} \mid \partial K_{0}\right)\right)$.

Proof. For the first part we need only show that for any compact $F \subset \partial U_{0} \mid \partial K_{1}$ we have $\lambda_{z}^{0} F=0$ if $\lambda^{*} F=0$. Since the harmonic measures for points in a given component of $K^{0}$ are equivalent, $\lambda^{*} F=0$ and our definition of $\lambda^{*}$ imply $\lambda_{z} F=0$ for every $z$ in $K^{0}$.

Since $F$ is a $G_{\delta}$. we can select a sequence $\left\{h_{n}\right\}$ in $C(\partial K)=C\left(\partial K_{0} \cup \partial K_{1}\right)$, all vanishing on a fixed neighborhood of $\partial K_{1}$, with $h_{n} \searrow \chi_{F}$ pointwise.

Let $\hat{h}_{n}^{0}$ be the continuous harmonic function $\left({ }^{9}\right)$ on $K_{0}$ which extends $h_{n} \mid \partial K_{0}$; $\hat{h}$ will denote the continuous harmonic function on $K$ which extends $h \in C(\partial K)$. Noting that $\partial K_{1}\left|\partial K_{0} \subset K\right| \partial K_{0} \subset K_{0} \mid \partial K_{0}=K_{0}^{0}$, we can set

$$
\begin{aligned}
k_{n} & =0 \text { on } \partial K_{0}, \\
& =\hat{h}_{n}^{0} \text { on } \partial K_{1} \mid \partial K_{0},
\end{aligned}
$$

to obtain a well-defined function on $\partial K=\partial K_{0} \cup \partial K_{1}$ which is continuous since $\hat{h}_{n}^{0} \rightarrow 0$ as we approach a point in $\partial K_{0} \cap \partial K_{1}$. Similarly

$$
\begin{aligned}
f & =0 \text { on } \partial K_{0}, \\
& =\lambda_{z}^{0} F \text { on } \partial K_{1} \mid \partial K_{0}
\end{aligned}
$$

defines an element of $C(\partial K)$ since $h_{n} \geqq \chi_{F} \geqq 0$ implies $\lambda_{z}^{0}\left(h_{n}\right)=\hat{h}_{n}^{0}(z) \geqq \lambda_{z}^{0} F \geqq 0, z \in K_{0}$, so that $f \rightarrow 0$ as we approach a point of $\partial K_{0} \cap \partial K_{1}$.

Now

$$
z \rightarrow \hat{h}_{n}^{0}(z)-\hat{k}_{n}(z)
$$

is a continuous harmonic function on $K$ with a restriction to $\partial K$ which is $h_{n}$ on $\partial K_{0}$ and $\hat{h}_{n}^{0}-\hat{h}_{n}^{0}=0=h_{n}$ on $\partial K_{1} \mid \partial K_{0}$, hence is $h_{n}$ on all of $\partial K=\partial K_{0} \cup \partial K_{1}$. So

$$
\hat{h}_{n}^{0}(z)-\hat{k}_{n}(z)=\hat{h}_{n}(z), \quad z \in K .
$$

As $n \rightarrow \infty, h_{n} \searrow \chi_{F}$ pointwise on $\partial K$, and so $\hat{h}_{n}(z)=\lambda_{z}\left(h_{n}\right) \searrow \lambda_{z} F, z \in K$, and

${ }^{(9)}$ I.e., continuous on $K_{0}$ and harmonic on $K_{0}^{0}$. Such an extension exists since $\partial K$ is the minimal boundary for $R(K)$; see the introductory remarks to this section. 
$\hat{h}_{n}^{0}(z)=\lambda_{z}^{0}\left(h_{n}\right) \searrow \lambda_{z}^{0} F, z \in K_{0}$ by monotone convergence. The latter implies $k_{n} \searrow f$ (defined in (3.8)), so that $\hat{k}_{n} \downarrow \hat{f}$, again by monotone convergence. By (3.9) then

$$
\lambda_{z}^{0} F-\hat{f}(z)=\lambda_{z} F=0, \quad z \in K^{0},
$$

so $\lambda_{z}^{0} F=\hat{f}(z)$ on $K^{0}$. Since $\hat{f}$ is continuous on $K$, and vanishes on $\partial K_{0}$ by (3.8), we conclude that

$$
\lim _{z \rightarrow z_{0}: z \in K^{0}} \lambda_{z}^{0} F=0, \quad z_{0} \in\left(\partial K_{0}\right) \cap K^{0-},
$$

so that for $z_{0}$ in a neighborhood $V$ of $F$ in $\partial U_{0} \mid \partial K_{1}\left(\subset\left(\partial K_{0}\right) \cap K^{0-}\right)$

$$
\lim _{z \rightarrow z_{0}: z \in K_{0}^{0}} \lambda_{z}^{0} F=0 .
$$

(Note that $K_{0} \cap W=K \cap W$ for a small neighborhood $W$ of $z_{0}$.)

On the other hand this also holds for $z_{0}$ in $\partial U_{0} \mid V$ since the harmonic extension to $K_{0}$ of any $g \geqq \chi_{F}$ in $C\left(\partial K_{0}\right)$ vanishing off $V$ tends to zero as we approach $z_{0}$. So the harmonic function $z \rightarrow \lambda_{z}^{0} F$ on $U_{0}$ tends to zero as we approach $\partial U_{0}$, and thus must vanish on all of $U_{0}$, in particular at $z \in U \subset U_{0}$, yielding our first assertion.

In the particular case that $\left(\partial U_{0} \mid \partial K_{1}\right) \cup\left(\partial U_{1} \mid \partial K_{0}\right) \subset \partial U$ the proof (starting now with $F \subset \partial U_{0} \mid \partial K_{1}$ having $\lambda_{z} F=0$ ) is unchanged up to (3.10), the latter part of which now only holds for $z$ in $U$. But replacing $K^{0}$ by $U$ the argument now leads to the same conclusion that $\lambda_{z}^{0} F \rightarrow 0$ as $z \rightarrow z_{0}$ from within $U_{0}$ (for $z_{0}$ in a neighborhood of $F$ in $\left.\partial U_{0} \mid \partial K_{1}\right)$ since $\partial U_{0} \mid \partial K_{1} \subset U^{-} \cap \partial K_{0}$ while $U \cap W=U_{0} \cap W$ for a small neighborhood $W$ of $z_{0}$. The remainder of the argument is unchanged.

LEMMA 3.10. Suppose the harmonic measure $\lambda=\lambda_{z_{0}}$ is a dominant representing measure on $R(K)$, while $(\operatorname{Re} R(K))_{\lambda}^{\perp}$ is at most one-dimensional and the component $U$ of $K^{0}$ containing $z_{0}$ is a part for $R(K)$ with $\partial U$ in the minimal boundary. Then

(a) $\lambda=\lambda_{z_{0}}$ is strongly dominant for $R(K)$ (hence for $A(K)$ as well), and $R(K)_{\lambda}^{\frac{1}{2}}$ $=A(K)_{\lambda}^{\perp}$.

(b) If $U$ is nicely connected (§1), $\lambda$ is the unique representing measure for $z_{0}$ on $R(K)$.

Note that if the conclusion of (a) holds for some $z_{0}$ in each Gleason part for $R(K)$, while $\partial K$ is the minimal boundary for $R(K)$, then 3.6 and 2.8 (or (2.3)) combine to show $R(K)=A(K)$.

To begin our proof of 3.10, by (2.3c) we have a strongly dominant representing measure for $z_{0}$, necessarily of the form $g \lambda$ for $g \in L^{1}(\lambda)$ since $\lambda$ is dominant. Since $\mathrm{Cg} \lambda \geqq \lambda$ for some constant $\mathrm{C}, \mathrm{g}$ is bounded below; to establish the first assertion of (a) we need only show $g$ is bounded. Of course we shall assume $g \not \equiv 1$, and since $\int g d \lambda=1, g>1$ and $g<1$ on sets of positive $\lambda$ measure, and $g-1$ cannot be of one sign.

Now $(g-1) \lambda$ is a real nonzero measure orthogonal to $R(K)$, hence spans $(\operatorname{Re} R(K))_{\lambda}^{\perp}$. So by Corollary 2.10 we have

$$
R(K)_{\lambda}^{\perp}=R(K)_{\delta \lambda}^{\perp}=\mathrm{C}(g-1) \lambda+H_{0}^{1}(g \lambda) \cdot g \lambda .
$$


We have $(g-1) r \lambda \in R(K)^{\perp}$ for $r \in R(K)$, and for $r(z) \equiv z$ by (3.11)

$$
z(g-1)=z_{1}(g-1)+f g, \quad z_{1} \in C, \quad f \in H_{0}^{1}(g \lambda),
$$

so $\left(z-z_{1}\right)(1-1 / g)=f \in H_{0}^{1}(g \lambda)$. So there are $r_{n}$ in $R(K), r_{n} \rightarrow\left(z-z_{1}\right)(1-1 / g)$ in $L^{1}(g \lambda)$ (hence also in $L^{1}(\lambda)$ ), and for $r \in R(K)$

$$
\int\left(1-\frac{1}{g}\right)\left(z-z_{1}\right) r d \lambda=\lim \int r_{n} r d \lambda=\lim \int r_{n} r g d \lambda=\int(g-1)\left(z-z_{1}\right) r d \lambda=0 .
$$

Thus $(1-1 / g) \lambda$ is orthogonal to $\left(z-z_{1}\right) \cdot R(K)$.

Of course if $z_{1} \notin K$ this implies the real measure $(1-1 / g) \lambda$ is orthogonal to $R(K)$, whence $(1-1 / g)$ is a multiple of $g-1: 1-1 / g=(g-1) / g=c(g-1)$. Since this implies $g=1$ or $g=1 / c$ a.e. $\lambda$ (so $g-1$ cannot change sign), we conclude that $z_{1} \in K$.

Now $(1-1 / g) \lambda$ is orthogonal to $\left(z-z_{1}\right) R(K)$, hence to any rational function in $R(K)$ vanishing at $z_{1}$. But any $r$ in $R(K)_{z_{1}}=\left\{r \in R(K): r\left(z_{1}\right)=0\right\}$ is the uniform limit of such rational functions, so that $(1-1 / g) \lambda$ is orthogonal to the maximal ideal $R(K)_{z_{1}}$. For the real number $k=-\lambda(1-1 / g)$ we thus have

$$
(1-1 / g) \lambda+k \lambda_{z_{1}}
$$

orthogonal to $C+R(K)_{z_{1}}=R(K)$.

If $z_{1}$ does not lie in the component $U$ of $K^{0}$ containing $z_{0}$, hence is not in the Gleason part containing $z_{0}$, then $\lambda_{z_{1}}$ and $\lambda=\lambda_{z_{0}}$ are mutually singular (else $\left.\left\|\lambda_{z_{1}}-\lambda\right\|<2\right)$, and so by $2.6(1-1 / g) \lambda \in R(K)^{\perp}$ so $(1-1 / g)=(g-1) / g=c(g-1)$, whence $g \equiv 1$ as earlier. And of course the same occurs if $k=0$, so we must have $k \neq 0$.

Thus $z_{1} \in U$, and the Radon-Nykodym derivative $h=d \lambda_{z_{1}} / d \lambda$ is bounded (as is $\left.h^{-1}=d \lambda / d \lambda_{z_{1}}\right)$ and satisfies

$$
(1-1 / g)+k h=c(g-1)
$$

for some real $c$, since (3.12) is now an element of $(\operatorname{Re} R(K))_{\lambda}^{\frac{1}{\lambda}}$. This shows $g$ is bounded since the left side of (3.13) is bounded while $c=0$ implies $(g-1) / g=-k h$ is of one sign, which is impossible if $g \not \equiv 1$. So we have the first assertion of (a).

For the second assertion, that $R(K)_{\lambda}^{\perp}=A(K)_{\lambda}^{\perp}$, we need only show $H^{2}(R(K), \lambda)$ $=H^{2}(A(K), \lambda)$ by Corollary 2.12 , since $\lambda$ is strongly dominant. But

$$
H^{2}(A(K), \lambda) \ominus H^{2}(R(K), \lambda)=H_{0}^{2}(A(K), \lambda) \ominus H_{0}^{2}(R(K), \lambda)
$$

is orthogonal in $L^{2}(\lambda)$ to $\bar{H}^{2}(A(K), \lambda)$, so to any element of $R(K)+\bar{R}(K)$, hence to $\operatorname{Re} R(K)$. The same of course applies to any element of

$$
\overline{H_{0}^{2}(A(K), \lambda)} \ominus \overline{H_{0}^{2}(R(K), \lambda)}
$$

which is the space of conjugates to elements of (3.14), and which is also orthogonal to (3.14). So if (3.14) is nonzero we have $(\operatorname{Re} R(K))_{\lambda}^{\frac{1}{\lambda}}$ a (complex) space of dimension at least two, showing (3.14) $=\{0\}$ and completing our proof of (a).

We obtain (b) by transporting matters to the unit disc. 
Since $U$ is nicely connected we have a Riemann map $\rho$ of the unit disc

$$
\{w \in C:|w|<1\}
$$

onto $U$ with $\rho(0)=z_{0}$, and a 1-1 map $\sigma$ defined on a subset of full measure of the unit circle which satisfies $\sigma\left(e^{i \theta}\right)=\lim _{r \rightarrow 1} \rho\left(r e^{i \theta}\right)$, with values in $\partial U$ of course. For a continuous harmonic function $\hat{f}$ on $U^{-}$we have

$$
\int \hat{f} d \lambda=\hat{f}(\rho(0))=\int \hat{f}\left(\rho\left(r e^{i \theta}\right)\right) \frac{d \theta}{2 \pi}=\lim _{r \rightarrow 1} \int \hat{f}\left(\rho\left(r e^{i \theta}\right)\right) \frac{d \theta}{2 \pi}=\int \hat{f}\left(\sigma\left(e^{i \theta}\right)\right) \frac{d \theta}{2 \pi}
$$

by dominated convergence, so

$$
\int f d \lambda=\int f \circ \sigma \frac{d \theta}{2 \pi}
$$

for any $f$ in $C(\partial U)$, hence for any bounded Baire function. Thus $f \rightarrow f \circ \sigma$ maps $L^{p}(\lambda)$ isometrically onto $L^{p}(\mu)$, where $d \mu=d \theta / 2 \pi$, while $g \rightarrow g \circ \sigma^{-1}$ provides the inverse, for all $p$.

Now let $H^{p}$ temporarily represent (the boundary value version of) the usual Hardy space on the unit circle. Since $f \circ \rho \in R(K) \circ \rho$ is a bounded analytic function, and $f \circ \sigma\left(e^{i \theta}\right)=\lim _{r \rightarrow 1} f\left(\rho\left(r e^{i \theta}\right)\right)$ on the domain of $\sigma, R(K) \circ \sigma \subset H^{\infty}$, and

$$
R(K) \subset H^{\infty} \circ \sigma^{-1} .
$$

Taking $L^{2}(\lambda)$ closures we obtain

$$
H^{2}(R(K), \lambda) \subset H^{2} \circ \sigma^{-1} .
$$

Now $\lambda$ is of course multiplicative on $H^{2} \circ \sigma^{-1}$ (by (3.17)) so, as earlier,

$$
\left(H^{2} \circ \sigma^{-1}\right) \ominus H^{2}(R(K), \lambda)=\left(H^{2} \circ \sigma^{-1}\right)_{0} \ominus H_{0}^{2}(R(K), \lambda)
$$

(where the noughts denote elements of mean zero) is orthogonal in $L^{2}(\lambda)$ to $\bar{H}^{2} \circ \sigma^{-1}$, hence to any element of $R(K)+\bar{R}(K)$, or to $\operatorname{Re} R(K)$. The same of course applies to

$$
\overline{\left(H^{2} \circ \sigma^{-1}\right)} \ominus \overline{H^{2}(R(K), \lambda)},
$$

the space of conjugates to elements of (3.20) and also orthogonal to (3.20). So (3.20) is nonzero only if we have $(\operatorname{Re} R(K))_{\lambda}^{\perp}$ at least two dimensional.

Thus we conclude that

$$
H^{2}(R(K), \lambda)=H^{2} \circ \sigma^{-1} .
$$

Since $g-1$ is bounded, and orthogonal to $R(K)$ in $L^{2}(\lambda)$, it is also orthogonal to (3.22), and for all $f$ in $H^{\infty}$

$$
0=\int\left(f \circ \sigma^{-1}\right)(g-1) d \lambda=\int f \cdot(g-1) \circ \sigma \frac{d \theta}{2 \pi}
$$


by (3.17). Consequently $(g-1) \circ \sigma=0$ a.e. on the circle, whence $g-1=0$ a.e. $\lambda$, completing our proof.

We can now proceed to our main applications to rational approximation. The first will be the cited result of Ahern and Sarason [AS 2] giving the rational defect when $S \backslash K$ has finitely many components. In [AS 2] this result was obtained from consequences of Ahern and Sarason's earlier detailed investigations of hypodirichlet algebras [AS 1]; aside from what we have developed so far our proof will use well-known facts valid when representing measures are unique, along with two relatively elementary and accessible facts given by Ahern and Sarason [AS 2, Lemmas 1-3]: if $S \backslash K$ has finitely many components, then points in distinct components of $K^{0}$ have mutually singular harmonic measures $\left({ }^{10}\right)$; moreover the simply connected components of $K^{0}$ are nicely connected.

We begin with the following special case of the Ahern-Sarason result, from which the general result will follow via 3.2.

Proposition 3.11 ([AS 2]). Suppose $S \backslash K$ has $n+1$ components $H_{0}, H_{1}, \ldots, H_{n}$, while each component of $K$ has a connected boundary (or, alternatively, each component of $K^{0}$ is simply connected). Then $R(K)$ is dirichlet on $\partial K$, and $A(K)=R(K)$.

Of course the second assertion follows from the first by 2.13 . We shall proceed by induction on $n$, assuming the result for $n-1$ (the case $n=0$ is of course Walsh's result [Wa 1]); by 3.4(f) we need only prove the first assertion assuming $K$ is connected.

Since $K$ is connected each "hole" $H_{i}$ is simply connected; and so has a connected boundary $[\mathrm{N}]$. Since $\partial K=\bigcup \partial H_{j}$ is connected by hypotheses, we have $\bigcup H_{i}^{-}$ connected, and a simple argument $\left({ }^{11}\right)$ shows we can find an $i$ for which $\bigcup_{j \neq i} H_{j}^{-}$ is connected; we shall take $i=0$, and so assume $\bigcup_{j \neq 0} H_{j}^{-}$, and thus $\bigcup_{j \neq 0} \partial H_{j}$, is connected.

Now set $K_{0}=S \backslash H_{0}, K_{1}=S \backslash \bigcup_{j \neq 0} H_{j}$, so that $\partial K_{\mathfrak{i}}$ is connected, $K=K_{0} \cap K_{1}$, $\partial K=\partial K_{0} \cup \partial K_{1}$, and, by our induction hypothesis, $R\left(K_{i}\right)$ is dirichlet on $\partial K_{i}$. Moreover $H_{0}$ is the union of an increasing sequence of simply connected domains $H_{0}^{v}$, with $H_{0}^{v-\subset H_{0}}$, so that $K$ is the intersection of a decreasing sequence $K^{v}=K_{1}$ $\cap K_{0}^{\nu}, K_{0}^{\nu}=S \backslash H_{0}^{\nu}$; since $\partial K_{0}^{\nu}=\partial H_{0}^{\nu}$ is connected, $R\left(K_{0}^{\nu}\right)$ is dirichlet on $\partial K_{0}^{\nu}$ so the rational defect for $K^{\nu}$ is $\leqq 1$ by 3.2 , and therefore the same is true for $K$ by sweeping, as in 3.1.

By 3.6 we have no completely singular measures orthogonal to $R(K)$, and if each representing measure $\lambda=\lambda_{z}$ is unique then by $[\mathrm{H} 1,6.7$, and $\mathrm{L}]$ there are no nonzero real measures in $R(K)_{\lambda}^{\perp}$, hence none in $R(K)^{\perp}$ by Theorem 2.8. So we can assume we have a $z$ in $K$ with a representing measure $\mu \neq \lambda_{z}$ carried by $\partial K$. Since the rational defect is at most $1, \sigma=\mu-\lambda_{z}$ must $\operatorname{span}(\operatorname{Re} R(K))^{\perp}$.

$\left({ }^{10}\right)$ Note that two such components can share the same boundary; cf. [K].

(11) Very similar to that used before 3.4(f). 
For our compact $K_{i}$ (above), let $\partial_{0}=\partial K_{0}=\partial H_{0}, \partial_{1}=\partial K_{1}=\bigcup_{j \neq 0} \partial H_{j}$. Since $R\left(K_{i}\right)$ is dirichlet on $\partial_{\mathfrak{i}}$, by sweeping we obtain as earlier $\left({ }^{12}\right)$ (cf. 3.2)

$$
\begin{aligned}
& \sigma_{\partial_{0} \mid \partial_{1}}+\int \lambda_{w}^{0} \sigma_{\partial_{1}}(d w)=0 \\
& \sigma_{\partial_{1}}+\int \lambda_{w}^{1} \sigma_{\partial_{0} \backslash \partial_{1}}(d w)=0
\end{aligned}
$$

so that $\sigma_{\partial_{0}} \mid \partial_{1} \neq 0($ else $\sigma=0)$ and

$$
\sigma_{\partial_{0} \mid \partial_{1}}=\int \eta_{t} \sigma_{\partial_{0} \mid \partial_{1}}(d t)
$$

where $\eta_{t}=\int \lambda_{w}^{0} \lambda_{t}^{1}(d w)$. So

$$
\sigma_{\partial_{0} \mid \partial_{1}}=\int\left(\eta_{t}\right) \partial_{0} \mid \partial_{1} \sigma_{\partial_{0} \mid \partial_{1}}(d t)
$$

and if $p$ is a unimodular function with $p \sigma_{\partial_{0}}\left|\partial_{1}=\right| \sigma_{\partial_{0}}\left|\partial_{1}\right|$ we have

$$
\left\|\sigma_{\partial_{0} \mid \partial_{1}}\right\|=\sigma_{\partial_{0} \mid \partial_{1}}(p)=\int\left(\eta_{t}\right)_{\partial_{0} \mid \partial_{1}}(p) \sigma_{\partial_{0} \mid \partial_{1}}(d t)
$$

so that, since $\left\|\eta_{t}\right\|=1,\left(\eta_{t}\right)_{\partial_{0} \mid \partial_{1}}(p)=1$ and $\eta_{t}\left(\partial_{0} \mid \partial_{1}\right)=1$ for some $t$ in $\partial_{0} \mid \partial_{1}$.

Now $H_{0}$ lies in one component $W$ of $K_{1}^{0}$, so that $\partial_{0}\left|\partial_{1} \subset H_{0}^{-}\right| \partial_{1} \subset W^{-} \mid \partial W=W$. Thus the second half of (3.23) implies $\partial W$ carries $\sigma_{\partial_{1}}$ since each $\lambda_{w}^{1}$ with $w \in \partial_{0} \mid \partial_{1}$ is carried by $\partial W$. Moreover our $t$ with $\eta_{t}\left(\partial_{0} \cap \partial_{1}\right)=0$ lies in $W$, so

$$
0=\int \lambda_{w}^{0}\left(\partial_{0} \cap \partial_{1}\right) \lambda_{t}^{1}(d w)
$$

But the closed support of $\lambda_{t}^{1}$ is precisely all of $\partial W$ (otherwise $W$ and some $H_{j}$, $j \neq 0$, lie in the same component $V$ of the complement of the support, and the analytic function $z \rightarrow \int((w-t) /(w-z)) \lambda_{t}^{1}(d w)$ on $V$ must vanish identically since it does on $H_{j}$; and of course it cannot vanish at $z=t$ ). So for each component $U$ of $K_{0}^{0}$ the relatively open subset $U \cap \partial W$ of the support of $\lambda_{t}^{1}$ contains a $w$ for which $\lambda_{w}^{0}\left(\partial_{0} \cap \partial_{1}\right)=0$ by (3.24), or is empty; since all $\lambda_{w}^{0}$ for $w$ in $U$ are equivalent, $\lambda_{w}^{0}\left(\partial_{0} \cap \partial_{1}\right)=0$ for all $w$ in $U \cap \partial W$, hence for all $w$ in $\partial W \backslash \partial_{0}$ since $\partial W \backslash \partial_{0} \subset K \backslash \partial_{0}$ $\subset K_{0}\left|\partial_{0}=K_{0}\right| \partial K_{0}=K_{0}^{0}$. As we saw $\partial W \mid \partial_{0}$ carries $\left.\sigma_{\partial_{1}}\right|_{0}$, and since

$$
\sigma_{\partial_{0}}+\int \lambda_{w}^{0} \sigma_{\partial_{1} \mid \partial_{0}}(d w)=0
$$

we conclude that for any Borel set $F \subset \partial_{0} \cap \partial_{1}$

$$
\sigma F=\sigma_{\partial_{0}}(F)=-\int \lambda_{w}^{0}(F) \sigma_{\partial_{1} \mid \partial_{0}}(d w)=-\int 0 \cdot \sigma_{\partial_{1} \mid \partial_{0}}(d w)=0
$$

So on $\partial_{0} \cap \partial_{1}, \sigma=0$, or $\mu=\lambda_{z}$.

${ }^{(2)} \lambda_{w}^{0}$ is harmonic measure relative to $K_{0}$; thus the first measure in (3.23) is a real measure on $\partial_{0}$ orthogonal to $R\left(K_{0}\right)$, etc. 
Now let $U$ be the component of $K^{0}$ containing our $z$ represented by $\mu$ and $\lambda_{z}$, and let $U_{i}$ be the component of $K_{i}^{0}$ containing $U, i=0,1$. Sweeping $\mu$ to $\partial_{i}=\partial K_{i}$ yields a measure representing $z$ on $R\left(K_{i}\right)$ (hence $=\lambda_{z}^{i}$ ) and necessarily $\geqq \mu_{\partial_{1}}$; thus $\mu \leqq \lambda_{z}^{0}+\lambda_{z}^{1}$. As a consequence, by Lemma $3.9, \mu$ is absolutely continuous with respect to the measure $\lambda^{*}$ on $\partial K \mid\left(\partial_{0} \cap \partial_{1}\right)=\left(\partial_{0} \mid \partial_{1}\right) \cup\left(\partial_{1} \mid \partial_{0}\right)$, and since $\mu=\lambda_{z}$ on $\partial_{0} \cap \partial_{1}$ as well, $\mu \ll \lambda^{*}$.

Now if $U$ is a Gleason part for $R(K)$ we have a contradiction to $\mu \neq \lambda_{z}$; for then $\mu$ is singular with respect to $\lambda_{w}$ for $w$ in any component of $K^{0}$ other than $U$, so $\mu \ll \lambda^{*}$ implies $\mu \ll \lambda_{z}$, and by $3.10(\mathrm{~b}), \mu=\lambda_{z}$ since $U$ is nicely connected.

So $U$ is not a Gleason part. If $\partial U \cap\left(\partial H_{j} \backslash \bigcup_{i \neq j} \partial H_{j}\right)=\varnothing$ for some $j$ we have a proper subset $F$ of $\left\{H_{0}, H_{1}, \ldots, H_{n}\right\}$ with $\partial U \subset \partial^{*}=\bigcup_{H \in F} \partial H$ and $\partial H \cap \partial U \neq \varnothing$ for $H$ in $F$, and if we set $K^{*}=S \backslash \bigcup_{H \in F} H$ then clearly $U$ cannot be an entire Gleason part for the subalgebra $R\left(K^{*}\right)$ of $R(K)$. On the other hand $\partial^{*}$ is connected since the simply connected sets $U, H_{j}$ all have connected boundaries, so $R\left(K^{*}\right)$ is dirichlet on $\partial^{*}$ by our induction hypotheses. But the nondegenerate Gleason parts for dirichlet algebras are connected by Wermer's theorem [We 2], and so are precisely the components of $K^{* 0} ; U$ is such a component, hence is a Gleason part for $R\left(K^{*}\right)$ and so for $R(K)$. We conclude then that $\partial U \cap\left(\partial H_{j} \mid \bigcup_{i \neq j} \partial H_{i}\right) \neq \varnothing$ for every $j$.

This implies $U \cup\left(\bigcup_{i \neq 0} H_{j}\right)$ lies in one component of $S \backslash H_{0}^{-}=K_{0}^{0}$ and the component is of course $U_{0}$. As a consequence if $x \in \partial U_{1}\left|\partial K_{0}=\partial U_{1}\right| \partial H_{0} \subset \bigcup_{j \neq 0} \partial H_{j} \mid \partial H_{0}$ then $x \in U_{0}^{-} \backslash \partial H_{0} \subset U_{0}$, so clearly $x \in \partial U$.

On the other hand $\partial U_{0} \mid \partial K_{1} \subset \partial U$ as well. For since $H_{0}$ lies in one component of $K_{1}^{0}$, either $H_{0} \subset U_{1}$, or $U_{1} \cap H_{0}^{-}=U_{1} \cap H_{0}=\varnothing$ so $U_{1} \subset S \backslash H_{0}^{-}=K_{0}^{0}$, whence $U_{1} \subset U_{0}$ and $U=U_{1}$. In the latter case $U$ is a component of $K_{1}^{0}$, hence a part for $R\left(K_{1}\right)$ which is again a contradiction. So $H_{0} \subset U_{1}$, and thus $\partial U_{0}\left|\partial K_{1} \subset \partial H_{0}\right| \partial K_{1} \subset U_{1}^{-} \mid \partial K_{1}$ $\subset U_{1}$. So clearly $\partial U_{0} \mid \partial K_{1} \subset \partial U$.

But now the last part of 3.9 says $\mu \leqq \lambda_{z}^{0}+\lambda_{z}^{1} \ll \lambda_{z}$ on $\partial U \backslash\left(\partial K_{0} \cap \partial K_{1}\right)=\partial U \mid\left(\partial_{0} \cap \partial_{1}\right)$ and since $\mu=\lambda_{z}$ on $\partial_{0} \cap \partial_{1}$, while $\partial U_{0} \cup \partial U_{1} \subset \partial U \cup\left(\partial_{0} \cap \partial_{1}\right)$ supports $\mu$, we have $\mu \ll \lambda_{z}$.

Since $U$ is not a Gleason part some $w$ in $K^{0} \mid U$ lies in the same part. Since we now know $\lambda_{z}$ is a dominant representing measure for $R(K)$, by $2.7 \lambda_{w} \ll \lambda_{z}$. This of course contradicts the result of Ahern and Sarason cited immediately preceding 3.11, and so completes our proof of 3.11.

Omitting the first three and last five paragraphs of the preceding proof (and replacing $H_{0}$ by $S \backslash K_{0}$ and $\bigcup_{j \neq 0} H_{j}$ by $S \backslash K_{1}$ ) yields a proof of the following.

Proposition 3.12. Suppose $K, K_{0}, K_{1}$ are compact and connected, with connected boundaries, $K=K_{0} \cap K_{1}, \partial K=\partial K_{0} \cup \partial K_{1}$, and $S \mid K_{0}$ lies in one component of $K_{1}^{0}$. Finally, suppose the components of $K^{0}$ are nicely connected and parts for $R(K)$, $R\left(K_{i}\right)$ is dirichlet on $\partial K_{i}, i=0,1$, and $(\operatorname{Re} R(K))^{\perp}$ is at most one-dimensional.

Then $R(K)$ is dirichlet on $\partial K$. 
Via Lemma 3.2 we can now pass easily from 3.11 to the full Ahern-Sarason result, of which the following is a slight variant.

THEOREM 3.13. Suppose $S \backslash K$ has finitely many components. Then $R(K)=A(K)$ and the nontrivial parts are precisely the components of $K^{0}$, while harmonic measures are strongly dominant. If $\lambda$ is the harmonic measure of a point in a component $U$ of $K^{0}$ then $(\operatorname{Re} R(K))_{\lambda}^{\frac{1}{\lambda}}$ is $n$-dimensional if $U$ is $n$-connected (i.e., has a boundary with $n+1$ components); in fact if $F_{0}, F_{1}, \ldots, F_{n}$ are the components of the boundary of $U$, $(\operatorname{Re} R(K))_{\lambda}^{\perp}$ is spanned by measures $f_{1} \lambda, \ldots, f_{n} \lambda$ where $f_{i}$ is a bounded function $\geqq 0$ on $F_{i}$, and $\leqq 0$ elsewhere, for $i=1, \ldots, n$.

Because of Remarks 3.4 we need only prove 3.13 when $K$ is connected, and then 3.4(f) and 3.11 combine to show the rational defect is at most one less than the number of components of $\partial K$; since the analytic defect is at least this large (cf. footnote 6), $(\operatorname{Re} R(K))^{\perp}$ and $(\operatorname{Re} A(K))^{\perp}$ have the same dimension, so $R(K)=A(K)$ by 2.11 .

Now choose a maximal set $F$ of components of $S \backslash K$ with the property that $\bigcup_{H \in F} H^{-}$is connected and set $C=\bigcup_{H \in F} H$. We shall prove 3.13 inductively assuming it holds for the compact set $K_{1}=K \cup C$.

Let $K_{0}=S \backslash C$, so $\partial K_{0}$ is connected and disjoint from $\partial K_{1}$, hence lies in one component $U_{1}$ of $K_{1}^{0}$, and $\partial K=\partial K_{0} \cup \partial K_{1}$. Evidently $\partial U_{1}$ separates $\partial K_{0}$ from $K_{1}^{0} \backslash U_{1}$, and thus $K_{1}^{0} \backslash U_{1}$ lies in one component $U_{0}$ of $K_{0}^{0}$. Now the components of $K^{0}$ are those of $K_{1}^{0}$ other than $U_{1}, U_{0} \cap U_{1}$, and those of $K_{0}^{0}$ other than $U_{0}$. So given two components of $K^{0}$ we see they lie in distinct Gleason parts by noting that they do for one of the subalgebras $R\left(K_{i}\right)$ of $R(K)$.

Thus the components of $K^{0}$ are the nontrivial parts, once we see each $z \in \partial K$ $=\partial K_{0} \cup \partial K_{1}$ is a peak point for $R(K)$; but $z$ is a peak point for $R\left(K_{i}\right) \subset R(K), i=0$ or 1 since we are assuming 3.13 for $K_{1}$ (and it holds for $K_{0}$ by 3.11).

For the same reason we can easily see $\lambda_{z}(z \in U)$ is dominant: by sweeping any representing measure $\mu$ to $\partial K_{1}$ we obtain a measure $\geqq \mu_{\partial K_{1}}$ which represents $z$ on $R\left(K_{1}\right)$, so that $\mu_{\partial K_{1}} \leqq C_{1} \lambda_{z}^{1}, \lambda_{z}^{1}$ being strongly dominant for $R\left(K_{1}\right)$. Similarly $\mu_{\partial K_{0}} \leqq C_{0} \lambda_{z}^{0}$, and (since $\partial U=\partial U_{0} \cup \partial U_{1}$ ) by $3.9 \lambda_{z}^{1}+\lambda_{z}^{0} \ll \lambda_{z}$, so $\mu \ll \lambda_{z}$. But we have no really direct proof that $\lambda_{z}$ is strongly dominant, and argue as follows.

As noted, the dimension $n$ of $(\operatorname{Re} R(K))^{\perp}$ is one less than the number of components of $\partial K$; if $U_{1}, \ldots, U_{k}$ are the (finitely many) components of $K^{0}$ which are multiply connected, and $U_{i}$ is $n_{i}$-connected, then $\sum_{i=1}^{k} n_{i}=n$ as is easily seen. So if we can produce, for each $i$, an independent set of $n_{i}$ bounded real elements of $L^{1}\left(\lambda_{z_{i}}\right)\left(z_{i} \in U_{i}\right)$ orthogonal to $R(K)$ we shall necessarily have a basis for $(\operatorname{Re} R(K))^{\perp}$, and then $(\operatorname{Re} R(K))_{\lambda_{z}}^{\perp}$ will always be spanned by finitely many bounded elements of $L^{1}\left(\lambda_{z}\right)$. Calling these $f_{1} \lambda_{z}, \ldots, f_{k} \lambda_{z}$ say, we have $\left(1 \pm \varepsilon f_{j}\right) \lambda_{z} \geqq 0$ for some $\varepsilon>0$ and all $j=1, \ldots, k$. Thus $\lambda_{z}$ (when not unique) can be realized as a relative interior point of the convex set $M_{z}$, hence is strongly dominant.

Such an independent set of $n_{i}$ bounded real elements $\mu_{1}, \ldots, \mu_{n_{i}}$ of $L^{1}\left(\lambda_{z_{1}}\right)$ is 
known, the natural set given by Ahern and Sarason [AS 1,2] and Wermer [We 4]: as a functional on $C\left(\partial U_{i}\right), \mu_{j}$ is given by

$$
\mu_{j}(f)=\int_{\gamma_{j}} \frac{\partial \hat{f}}{\partial n} d s
$$

where $\left({ }^{13}\right) \hat{f}$ is the continuous harmonic extension to $U_{i}^{-}$of $f$ and $\gamma_{1}, \ldots, \gamma_{n_{t}}$ is a homology basis of smooth curves in $U$.

For the final assertion we need only note that for any of our $U_{i}$ no component of $\partial U_{i}$ separates the remainder, so that exactly the argument of $3.4(\mathrm{~g})$ applies to yield a basis of the prescribed special kind. This completes our proof of 3.13.

The preceding proof applies equally well to show the following.

Proposition 3.14. Suppose $K=\bigcap_{i=0}^{n} K_{i}$, where the compact $K_{i}$ have pairwise disjoint connected boundaries covering $\partial K$ and $R\left(K_{i}\right)$ is dirichlet on $\partial K_{i}, i=0,1, \ldots, n$. Then the rational defect for $K$ is $n$, components of $K^{0}$ form the nontrivial parts, harmonic measures are strongly dominant, etc., as in 3.13.

The methods developed so far yield a variety of $K$ with infinitely many complementary components for which rational approximation holds. There are of course trivial examples; as earlier 3.4(d) and Krě̀n-Milman reduce showing $R(K)=A(K)$ to showing it for each component, so one trivial instance is that in which $S \backslash F$ has finitely many components for each component $F$ of $K$. Less trivially, $R(K)=A(K)$ if $\partial K$ is covered by the boundaries of finitely many holes: for then in the notation of 3.6, $K=K_{Q}^{*}$ for a $K^{*}$ to which 3.13 applies, so one concludes from $R(K)^{\perp}$ $=\sum_{\Lambda} R\left(K^{*}\right)_{\lambda}^{\perp}$ that the rational defect for $K$ is at most the sum of the connectivities of the multiply connected components of $K^{0}$, and that is at least the analytic defect, as in 3.13, so both coincide. But we can in fact build much more complicated examples.

LeMma 3.15. Let $K=K_{\infty} \cap\left(\bigcap_{n=1}^{\infty} K_{n}\right)$, where $\left\{K_{n}\right\}$ is a decreasing sequence of compact connected sets in $C$ and $\partial K_{n} \subset \partial K_{n+1}$ (so the components of $S \backslash K_{n}$ form a subset of those of $S \backslash K_{n+1}$, hence of $\left.S \backslash K\right)$, while $\partial K_{n} \cap \partial K_{\infty}=\varnothing$ for all $n$. Let $E$ be the set of all $z$ in $\partial K$ with the property that each of its neighborhoods meets infinitely many of the sets $\partial K_{n+1} \mid \partial K_{n}$. Suppose

(a) $\partial K=\bigcup_{n=1}^{\infty} \partial K_{n} \cup \partial K_{\infty} \cup E$, and is connected.

$\left({ }^{13}\right)$ The integral (where $d s$ is the element of arc length) gives the period of the conjugate harmonic function, hence vanishes when that function is single valued, so $\mu_{\mathrm{t}}$ is orthogonal to $\operatorname{Re} R(K)$; that $\mu_{1}, \ldots, \mu_{n_{1}}$ are independent is easily seen and that $\mu_{j}$ is a bounded functional is a well-known consequence of the smoothness of the Green's function when $U_{i}$ is bounded by analytic curves [Ne], and if $\tilde{U}_{i} \subset U_{i}$ contains our $\gamma_{1}, \ldots, \gamma_{n_{i}}$ and is bounded by analytic curves we may obtain our $\mu_{f}$ as swept versions of those we obtain for $\tilde{U}_{:}$. This suffices to provide the

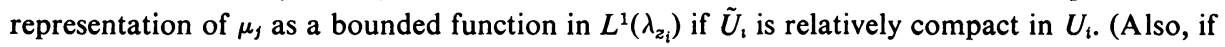
each $\gamma_{i}$ is chosen to enclose precisely one component of $\partial U,\left\{\mu_{j}\right\}$ has the final property stated in 3.13 , as is easily seen.) 
(b) $R\left(K_{n}\right)$ is dirichlet on $\partial K_{n}, n=1,2, \ldots$ (and for $n=\infty$ as well if $K_{\infty} \neq S$ ), and each boundary is connected.

(c) E is a peak interpolation set $\left({ }^{14}\right)$.

(d) Any two components of $K^{0}$ lie in distinct Gleason parts for $R\left(K^{*}\right)$ for some $K^{*} \supset K$.

(e) Each component of $K^{0}$ is nicely connected.

Then $R(K)$ is dirichlet on $\partial K$.

As an example of the application of 3.15, consider removing the interior of an infinitely branching tree of tangent closed discs from an annulus $R$ as follows: starting with a root disc tangent to the outer bounding circle, remove a sequence of open discs proceeding upwards, branching to form a $Y$, the branches again branching etc., with the top of the tree moving towards (and accumulating on) the inner bounding circle of $R$. Suppose the leftmost route along the branches ("the left branch") leads to a single point $z_{0}$ of the inner bounding circle of $R$.

Now 3.15 does not apply directly: (d) will be hard to verify a priori, while (c) need not hold. But we need not apply 3.15 directly; the set $K$ we obtain is the intersection of a sequence $\left\{K_{v}\right\}$, where $K_{v}$ has all but "the left branch" pruned, i.e., where all branches end $\nu$ discs from the root disc except for our path of discs leading to $z_{0}$. So (cf. 3.1) we need only see each $K_{v}$ has $R\left(K_{v}\right)$ dirichlet, and 3.15 easily applies to $K=K_{v}: E=\left\{z_{0}\right\}$, and if two distinct components of $K^{0}$ appear, one is bounded by finitely many holes so a set $K^{*}$ (to which 3.13 applies) is available as in (d).

Now suppose the branches do not accumulate at all points of the inner boundary of $R$, and let $U$ be the union of the bounded components of $S \backslash K$. Choose a small replica $U_{1}$ of $U$ lying in one component of $K^{0}$ with $\partial U_{1} \cap \partial U$ a single point $z_{1}$ of the inner boundary of $R$. Taking $K_{0}=S \backslash U_{1}, K_{1}=K$ it is easy to see $\operatorname{Re}\left(R\left(K_{0} \cap K_{1}\right)\right)^{\perp}$ is at most one-dimensional (removing a small neighborhood of $z_{1}$ from $U_{1}$ yields an altered $K_{0}$ with $\partial K_{0} \cap \partial K=\varnothing$, so 3.2 applies; now apply 3.1). Thus 3.12 shows $R\left(K_{0} \cap K_{1}\right)=R\left(K \backslash U_{1}\right)$ is dirichlet on $\partial\left(K \backslash U_{1}\right)$. Evidently we can now grow our "tree" in $R$ using replicas of $U$ instead of discs.

Proof of 3.15. Since $R(K) \mid E=C(E)$, each $z \in E$ is a peak point of $R(K) \mid E$, and since $E$ is a peak set, $z$ is a peak point of $R(K)$ by a result of Bishop [B 2]. Thus $\partial K$ is the minimal boundary of $\bar{R}(K)$ by (a) and (b).

So each nontrivial part lies in $K^{0}$ and since a part for $R(K)$ is contained in one part for $R\left(K^{*}\right)$ when $K^{*} \supset K$, (d) implies the components $U$ of $K^{0}$ are the nontrivial parts.

Now if $z \in U$ and $\mu \in M_{z}$ we have $\mu E=0$ since if $f$ peaks on $E,|f(z)|<1$, so $\mu E=\lim \mu\left(f^{n}\right)=0$. Thus we can see $\lambda_{z}$ is dominant by showing $\mu \ll \lambda_{z}$ on $\partial K \backslash E$, hence on any compact subset $F$ of $\partial K \backslash E$. Choose an open neighborhood $V$ of $F$

${ }^{(14)}$ That is, $E$ is a peak set for which $R(K) \mid E=C(E)$. 
disjoint from $E$; by compactness we have an $n$ for which $F \subset \partial K_{n} \cup \partial K_{\infty}$ and $V \cap\left(\partial K_{k+1} \mid \partial K_{k}\right)=\varnothing$ for $k \geqq n$, so $V \cap\left(\partial K_{n+m} \mid \partial K_{n}\right)=\varnothing, m \geqq 1$. In (almost) the notation of 3.9, we set $K_{0}^{*}=K_{n} \cap K_{\infty}$ (so $S \backslash K_{0}^{*}$ consists of the components of $S \backslash K$ lying outside $K_{0}^{*}$ ), and take $K_{1}^{*}$ as the complement of the union of the remaining components of $S \backslash K$, so $K=K_{0}^{*} \cap K_{1}^{*}, \partial K=\partial K_{0}^{*} \cup \partial K_{1}^{*}=\partial_{0} \cup \partial_{1}$. Now sweeping $\mu$ to $\partial_{0}=\partial K_{0}^{*}$ yields a measure $\geqq \mu_{\partial_{0}}$ which represents $z$ on $R\left(K_{0}^{*}\right)$, hence is bounded by $c \lambda_{z}^{0}$ since $\lambda_{z}^{0}$ is strongly dominant for $R\left(K_{0}^{*}\right)=R\left(K_{n} \cap K_{\infty}\right)$ by 3.14 . So by 3.9 $\mu \ll \lambda_{z}^{0} \ll \lambda^{*}$ on $\partial K_{0}^{*} \mid\left(\partial_{0} \cap \partial_{1}\right)$, in particular on $F$ which lies in this set since $V \cap \partial_{1}=\varnothing$. Since we have seen that the components of $K^{0}$ are parts, $\mu$ is singular with respect to $\lambda_{w}, w \notin U$, so $\mu \ll \lambda^{*}$ on $F$ implies $\mu \ll \lambda_{z}$ on $F$ and $\lambda_{z}$ is dominant.

Now we know $\partial K$ is the minimal boundary for $R(K)$, the components of $K^{0}$ are nicely connected and parts, and harmonic measures are dominant, so if $(\operatorname{Re} R(K))_{\lambda}^{\perp}$ is at most one-dimensional for $\lambda=\lambda_{z}, z \in K^{0}$, then by $3.10(\mathrm{~b})$ representing measures are unique, which implies our result as we saw in 3.11. But $(\operatorname{Re} R(K))_{\lambda}^{\frac{1}{\lambda}}$ is carried by $\partial K_{\infty} \cup \bigcup_{n=1}^{\infty} \partial K_{n}$ since $\lambda E=0$, and if that space is of dimension greater than one, the same is true for the space $N$ of real measures orthogonal to $R\left(K_{n} \cap K_{\infty}\right)$ we obtain by sweeping our measures to $\partial K_{\infty} \cup \partial K_{n}$ $=\partial\left(K_{\infty} \cap K_{n}\right)$ for some $n$ by 3.1 ; but since $\partial K_{\infty} \cap \partial K_{n}=\varnothing, 3.2$ implies $N$ is at most one-dimensional, so that $(\operatorname{Re} R(K))_{\lambda}^{\frac{1}{\lambda}}$ is also, and our proof is complete.

It should be noted how our last two results combine to yield further examples where $R(K)=A(K)$. These all have only finitely many multiply connected components of $K^{0}$, and the rational defect is finite. To arrive at examples with infinite rational defect we need

Lemma 3.16. Suppose $z_{0}$ lies in a component of $K^{0}$ which is a part for $R(K)$, and $\lambda$ is the harmonic measure for $z_{0}$. Then in $L^{2}(\lambda), E=H^{2}(A(K)) \ominus H^{2}(R(K))$ is finite dimensional only if $E=\{0\}$.

Proof. Suppose $E \neq\{0\}$ and let $g_{1}, \ldots, g_{n}$ be an orthonormal basis for $E$. Since $H^{2}(A(K)$ ) is an $R(K)$-module (which, as the reader will note, is almost the only property of $H^{2}(A(K))$ we shall use) for $g \in E$ and $r \in R(K) r g=g^{\prime}+r^{\prime}$ with $g^{\prime} \in E$ and $r^{\prime} \in H^{2}(R(K))$ unique, so $T_{r} g=g^{\prime}$ defines a representation $T$ of $R(K)$ on $E$. Since the representation is finite dimensional and $R(K)$ abelian we have a common eigenvector which we can take as $g_{1}$. Thus $T_{r} g_{1}=\phi(r) \cdot g_{1}$, or $r g_{1}=\phi(r) g_{1}+r^{\prime}$, where $\phi(r) \in C$ and $r^{\prime} \in H^{2}(R(K))$. Trivially $\phi$ is a multiplicative linear functional, 1 at 1 , and so corresponds to evaluation at $z_{1} \in K$. And

$$
\begin{aligned}
\left|g_{1}\right|^{2} \lambda(r) & =\bar{g}_{1} \lambda\left(r g_{1}\right)=\bar{g}_{1} \lambda\left(\phi(r) g_{1}+r^{\prime}\right) \\
& =\phi(r) \lambda\left(\left|g_{1}\right|^{2}\right)+\bar{g}_{1} \lambda\left(r^{\prime}\right)=\phi(r) \cdot 1+0=r\left(z_{1}\right)
\end{aligned}
$$

since $g_{1}$ is orthogonal to $H^{2}(R(K))$ in $L^{2}(\lambda)$. This shows $z_{1}$ lies in the same part as $z_{0}$, hence in the same component of $K^{0}$, and $\lambda_{1}=\lambda_{z_{1}}$ is boundedly equivalent to $\lambda$. 
In particular then $\lambda_{1}$ yields a continuous functional $\left({ }^{15}\right)$ on $L^{2}(\lambda)$, and one which is multiplicative on the $L^{2}(\lambda)$ - (or $L^{2}\left(\lambda_{1}\right)$-) closure of $A(K)$. So since $\phi(r)=z_{1}$ for $r(z) \equiv z, z g_{1}=z_{1} g_{1}+r^{\prime}, r^{\prime} \in H^{2}(R(K))$, which implies

$$
\lambda_{1}\left(r^{\prime}\right)=\lambda_{1}\left(z-z_{1}\right) \cdot \lambda_{1}\left(g_{1}\right)=0 \cdot \lambda_{1}\left(g_{1}\right)=0 .
$$

But $r \rightarrow \lambda_{1}(r)=r\left(z_{1}\right)$ is continuous on $R(K)$ in the $L^{2}(\lambda)$ norm, so that if $r_{v}$ in $R(K)$ converges to $r^{\prime}$ in $L^{2}(\lambda), r_{v}\left(z_{1}\right) \rightarrow \lambda_{1}\left(r^{\prime}\right)=0$, and we may just as well take $r_{v}\left(z_{1}\right)=0$. Thus $r_{v} /\left(z-z_{1}\right) \in R(K), r_{v} /\left(z-z_{1}\right) \rightarrow r^{\prime} /\left(z-z_{1}\right)=g_{1}$ in $L^{2}(\lambda)$, and we find $g_{1} \in H^{2}(R(K))$, our contradiction $\left({ }^{16}\right)$.

The fact that the proof of 3.16 applies with $H^{2}(A(K))$ any $R(K)$-module on which $\lambda_{z_{1}}$ is multiplicative allows us to draw the following consequence, which even here is weaker than the result obtained by Ahern and Sarason [AS 1, 14.5] for hypo-dirichlet algebras when $R(K)$ is such an algebra.

COROLlaRY 3.17. Suppose $z_{0}$ lies in a component $U$ of $K^{0}$ which is a part for $R(K)$, the harmonic measure for $z_{0}$ is $\lambda=\lambda_{z_{0}}$, and in $L^{2}(\lambda)$ the subspace orthogonal to $R(K)+\bar{R}(K)$ is finite-dimensional. Then $H^{\infty}(R(K), \lambda)$ is maximal among the subalgebras of $L^{\infty}(\lambda)$ on which $\lambda_{z}$ is multiplicative for every $z$ in $U$.

Indeed if $B \supset H^{\infty}(R(K))$ is such an algebra then since $\lambda$ is multiplicative on $B \supset R(K), B$ is orthogonal in $L^{2}(\lambda)$ to $\bar{R}(K)_{z_{0}}$ and thus, if $H^{2}(B)$ is the closure of $B$ in $L^{2}(\lambda), E=H^{2}(B) \ominus H^{2}(R(K))$ is orthogonal to $R(K)+\bar{R}(K)_{z_{0}}=R(K)$ $+\bar{R}(K)$, hence finite-dimensional. By the proof of 3.16 we conclude that $E=\{0\}$, so each $f \in B$ lies in $H^{2}(R(K)) \cap L^{\infty}=H^{\infty}(R(K))$. Except for the possibility that no such algebra $B$ may exist, we are done; but as noted after $2.14, B=H^{\infty}(R(K))$ is one.

We have noted 3.17 since it suffices to yield the consequence of [AS 1, 14.5] Ahern and Sarason obtain in [AS 2, Theorem 2] by exactly their proof.

If $U$ is an $n$-connected component of $K^{0}$ it is the conformal image of a domain $D$ bounded by $n+1$ disjoint circles [Ne]; if the corresponding map $\rho$ has nontangential boundary values which yield a 1-1 map $\rho^{*}$ on almost all of $\partial D$ (relative to harmonic measure $\mu$ for any point $w$ in $D$ ), as in [AS 2, §3], we shall say $U$ is nicely n-connected. This holds in particular if $S \backslash K$ has finitely many components [AS 2, §3], and allows

(15) If $g_{1}$ were bounded, $r \rightarrow\left|g_{1}\right|^{2} \lambda(r)=r\left(z_{1}\right)$ would extend to a continuous functional on $L^{2}(\lambda)$; and so (without assuming $\lambda$ is the harmonic measure and $U$ a part, but using $\left|g_{1}\right|^{2} \lambda$ in place of $\lambda_{1}$ ) the proof leads again to a contradiction. Thus if $\lambda$ is any representing measure on $R(K)$ and $E=H^{2}(A(K), \lambda) \ominus H^{2}(R(K), \lambda)$ is finite dimensional and consists of bounded functions, $E=\{0\}$.

${ }^{\left({ }^{16}\right)}$ A similar argument shows that whenever $\partial K$ is the minimal boundary for $R(K)$, $R(K)$ cannot have finite positive defect in $A(K)$. (Take a finite-dimensional complementary subspace $E$ of $R(K)$ in $A(K)$; one again gets a representation $T$ and a common eigenvector $g$ in $E$, and $r g=r\left(z_{1}\right) g+r^{\prime}, r^{\prime} \in R(K)$. Choosing a measure $\mu$ on $\partial K$ orthogonal to $R(K)$ with $\mu(g)=1, \mu(r g)=r\left(z_{1}\right)$; since $z \in \partial K$ is a peak point, $\mu\{z\}=0$ for each $z \in \partial K$, so $z_{1} \notin \partial K$. Thus $\left(z-z_{1}\right) g=r^{\prime}$ implies $r^{\prime}\left(z_{1}\right)=0$ and so $g \in R(K)$.) 
one to obtain the measure space associated with $\partial U$ and $\lambda_{\rho(w)}$ as the transported version of that associated with $\partial D$ and $\mu$. And in this situation as in [AS 2, §3] we can transplant the boundary value functions of $\mathfrak{S}(D)$, the space of bounded analytic functions on $D$, to yield an algebra $B \supset H^{\infty}\left(R(K), \lambda_{\rho(w)}\right)$ on which $\lambda_{\rho(w)}$ is multiplicative; thus by exactly the proof of [AS 2, Theorem 2] using 3.17 in place of Ahern and Sarason's Lemma 4, we obtain

COROLlaRY 3.18 [AS 2]. Suppose $z_{0}$ lies in a nicely n-connected component $U$ of $K^{0}$ which is a part for $R(K)$, and in $L^{2}\left(\lambda_{z_{0}}\right)$ the subspace orthogonal to $\operatorname{Re} R(K)$ is finite dimensional. Then if $\mathfrak{S}(U)$ is the (sup-normed) space of bounded analytic functions on $U, H^{\infty}\left(R(K), \lambda_{z_{0}}\right)$ is isometrically isomorphic to $\mathfrak{S}(U)$ under the map $f \rightarrow \hat{f}$, where $\hat{f}(z)=\lambda_{z}(f), f \in H^{\infty}\left(R(K), \lambda_{z_{0}}\right)$.

We proceed to our main consequence of 3.16.

Thговем 3.19. Suppose: (1) the minimal boundary for $R(K)$ covers all of $\partial K$ except for a set of plane measure zero (i.e., that $R(\partial K)=C(\partial K)$ by Bishop's theorem [B 2]) and (2) for each component $U$ of $K^{0}$ there is a compact $K_{U} \supset K$ having $U$ as a component of $K_{U}^{0}$ and a part for $R\left(K_{U}\right)$, while the harmonic measure $\lambda_{z}$ for $z \in U$ is strongly dominant for $R\left(K_{U}\right)$ and $\left(\operatorname{Re} R\left(K_{U}\right)\right)_{\lambda_{z}}^{\perp}$ is finite dimensional.

Then $R(K)=A(K)$, the nontrivial parts are the components of $K^{0}$, and harmonic measures are strongly dominant.

In particular, if (1) holds and each component $U$ of $K^{0}$ is bounded by finitely many holes, $R(K)=A(K)$ since $K_{U}$ can be taken as the complement in $S$ of the union of those holes, and 3.13 applies to $K_{U}$.

To proceed to the proof of 3.19, note first that $U$, as a part for $R\left(K_{U}^{\prime}\right) \subset R(K)$, is a part for $R(K)$. So $K^{0}$ is a union of parts and each $z \in \partial K$ lies in a part contained in $\partial K$. By a recent result $\left({ }^{17}\right)$ of Wilken [Wi], this and (1) imply $z$ is itself a part and in fact a peak point for $R(K)$. So $\partial K$ is the minimal boundary for $R(K)$, and the nontrivial parts are precisely the components of $K^{0}$.

Let $U$ be a component of $K^{0}, z \in U$. Since $U$ is also a component of $K_{U}$ the harmonic measure $\lambda_{z}$ relative to either $K$ or $K_{U}$ is the same. Moreover $\mu \in M_{z}(R(K))$, when swept to a measure $\mu^{\prime}$ on $K_{U}$ representing $z$ on $R\left(K_{U}\right)$ has $\mu^{\prime} \leqq C_{z} \cdot \lambda_{z}$ since $\lambda_{z}$ is strongly dominant for $R\left(K_{U}\right)$. But evidently $\mu=\mu^{\prime}$ (so that $M_{z}(R(K)) \subset$ $M_{z}\left(R\left(K_{U}\right)\right)$ and $\lambda_{z}$ is strongly dominant for $\left.R(K)\right)$ : for we have a Borel set $E \subset \partial U$ carrying $\lambda_{z}$ (and so $\mu^{\prime}$ ) and of measure zero $\left({ }^{18}\right)$ for $\lambda_{w}^{K}, w \in K_{U} \backslash U^{-}$, so that

$$
\mu^{\prime}=\mu_{\partial U}+\int_{\partial K \backslash U^{-}} \lambda_{w}^{K} \mu(d w)
$$

implies $1=\mu^{\prime}(E)=\mu_{\delta U}(E)+0$, and $\mu^{\prime}=\mu_{\partial U}=\mu$.

$\left({ }^{17}\right)$ Which states that $z \in K$ is either a peak point for $R(K)$ or lies in a part with positive plane measure (for any $K$ ).

$\left({ }^{18}\right)$ By Wilken's result there are only countably many non-peak-point-parts for $R\left(K_{U}\right)$ so we only need to insure $E^{\prime}$ supports countably many measures singular with respect to $\lambda_{z}$; for peak points $w$ in $K_{U} \backslash U^{-}, \lambda_{w}^{K} U(E)=\delta_{w}(E)=0$ automatically. 
By 3.6 we have no completely singular orthogonal measures, and to prove $A(K)=R(K)$ we need only show $A(K)_{\lambda_{z}}^{\perp}=R(K)_{\lambda_{z}}^{\perp}$ for all $z$ in $K^{0}$. Taking $z \in U$ as above, by 2.12 we need only see the $L^{2}\left(\lambda_{z}\right)$-closures of $A(K)$ and $R(K)$ coincide since $\lambda_{z}$ is strongly dominant.

But $E=H^{2}(A(K)) \ominus H^{2}(R(K))$ lies in the subspace of $L^{2}\left(\lambda_{z}\right)$ orthogonal to $\operatorname{Re} R(K)$, so $E \cdot \lambda \subset(\operatorname{Re} R(K))_{\lambda_{z}}^{\perp} \subset\left(\operatorname{Re} R\left(K_{U}\right)\right)_{\lambda_{z}}^{\perp}$ which is finite dimensional, and 3.16 applies to show $E=\{0\}$, completing our proof.

Some final comments are in order on the components $R(K)_{\lambda}^{\perp}$ of the decomposition (2.3) we obtain for those $K$ which we can construct via $3.14,3.15,3.19$. When $K$ is the minimal boundary, components are parts and harmonic measures are dominant, the final assertion of 3.6 shows that each component $R(K)_{\lambda}^{\perp}$ is precisely $R\left(U^{-}\right)^{\perp}$ for the corresponding component $U$ of $K^{0}$. And whenever $U$ is a nicely $n$-connected component of $K^{0}$ which is a part, while the harmonic measure $\lambda_{z}$ of one of its points is strongly dominant then $R(K)_{\lambda_{z}}^{\perp}$ is merely the transported version of a space $R(B)^{\perp}$, where $B$ is the closure of a domain bounded by finitely many disjoint circles conformally equivalent to $U$.

Indeed if $\rho$ is the conformal map of $B^{0}$ onto $U$, then we know the measure space associated with $\partial U$ and $\lambda_{z}$ is that associated with $\partial B$ and $\lambda_{\rho}^{B}{ }^{-1}(z)=\mu$ transported via the map $\rho^{*}$, and then $H^{\infty}(\mu)$ corresponds precisely to $H^{\infty}\left(\lambda_{z}\right)$ since $\left(H^{\infty}\left(\lambda_{z}\right) \circ \rho^{*}\right)^{\wedge}$ $=H^{\infty}\left(\lambda_{z}\right)^{\wedge} \circ \rho=\mathfrak{E}(U) \circ \rho=\mathfrak{E}\left(B^{0}\right)=H^{\infty}(\mu)^{\wedge}$, as is easily verified (using 3.18 of course), where, as there, $\hat{f}(w)=\lambda_{w}(f)$, etc. Since $f \rightarrow \hat{f}$ is an isomorphism, $H^{\infty}\left(\lambda_{z}\right) \circ \rho^{*}$ $=H^{\infty}(\mu)$. But the elements of $R(K)_{\lambda_{z}}^{\perp}$, or of $R(B)_{\mu}^{\perp}=R(B)^{\perp}$, are just those of $L^{1}\left(\lambda_{z}\right)$ or $L^{1}(\mu)$ orthogonal to $H^{\infty}\left(\lambda_{z}\right)$ or $H^{\infty}(\mu)$ (by 2.9), so $H^{\infty}\left(\lambda_{z}\right) \circ \rho^{*}=H^{\infty}(\mu)$ implies $\rho^{*} R(B)^{\perp}=R(K)_{\lambda_{z}}^{\perp}$. (In particular then, the real orthogonal measures are given by bounded functions in $L^{1}\left(\lambda_{z}\right)$.)

4. Interpolation as an application. Let $K$ again be a compact plane set, and let $\lambda^{*}=\sum_{n=1}^{\infty} 2^{-n} \lambda_{n}$ where $\lambda_{n}$ is the harmonic measure of a point in the $n$th component of $K^{0}$ as in 3.9. In those cases in which we have been able to show $R(K)$ $=A(K)$ in the preceding section, we have also obtained considerable information on $A(K)^{\perp}$ (cf. 2.8, 2.10,3.6); in particular it lies in $L^{1}\left(\lambda^{*}\right)$, and as an immediate consequence we know the closed subsets $F$ of $\partial K$ for which $A(K)$ interpolates $C(F): A(K) \mid F=C(F)$ iff $\lambda^{*} F=0$. (Indeed if $\lambda^{*} F=0$ (and $A(K)^{\perp} \subset L^{1}\left(\lambda^{*}\right)$ ) then $\mu_{F}=0$ for each $\mu$ in $A(K)^{\perp}$, so our conclusion follows by [Gl $1,4.8$ ], which in fact shows $F$ is a peak set as well. On the other hand if $\lambda_{z} F>0$ for some $z$ in some component $U$ of $K^{0}$ then this inequality holds for all $z$ in $U$. Choose $f \in C(F)$ which doesn't vanish at some $z_{0} \in F \cap U^{-}$, but does vanish on a subset of $F$ positive $\lambda_{z}$-measure (for some, hence all, $z$ in $U$ ). If $g \in A(K)$ has $g \mid F=f$ then $g(z)=\lambda_{z}(g)=0$ for $z$ in $U$ by Jensen's inequality, so $g \mid U^{-}=0$ and $f\left(z_{0}\right)=0$. Thus $\lambda^{*} F=0$ if $A(K) \mid F=C(F)$.)

What we want to point out in the present section is that the subsets of $K$ (rather than just $\partial K$ ) which are sets of interpolation can essentially be determined in the same setting. 
Let $\mathfrak{S}_{(}(U)$ be the sup-normed space of bounded analytic functions on the open set $U \subset S$, and for $F \subset U$ say $\mathfrak{S}(U)$ interpolates $C(F)$ with bound $M$ if, for each $f$ in $C(F)$, there is a $g$ in $\mathfrak{S}(U)$ with $g \mid F=f,\|g\| \leqq M\|f\|$. (Of course $F$ must be given by a sequence with no cluster points in $U$.)

THEOREM 4.1. Suppose $\partial K$ is the minimal boundary for $R(K)$; each component $U$ of $K^{0}$ is nicely $n$-connected and a part for $R(K)$ with $\lambda=\lambda_{z}(z \in U)$ strongly dominant; finally that the subspace of $L^{2}(\lambda)$ orthogonal to $\operatorname{Re} R(K)$ is finite dimensional.

Let $F$ be a closed subset of $K$. Then $\left({ }^{19}\right) A(K)=R(K)$ interpolates $C(F)$ iff

(a) $\lambda^{*}(F \cap \partial K)=0$, and

(b) there is a constant $M$ for which $\mathfrak{S}_{(}(U)$ interpolates $C(F \cap U)$ with bound $M$ for each component $U$ of $K^{0}$.

(Note that the second hypothesis holds if each $U$ is bounded by finitely many components of $S \backslash K$.) Thus interpolation by $A(K)$ reduces, on $K^{0}$, to interpolation by bounded analytic functions (with some uniformity as we pass among the components of $K^{0}$ ), exactly as in the case of the unit disc [H 2]. If $U$ is an $n$-connected component of $K^{0}$, and we divide $F \cap U$ into $n+1$ sequences $F_{i}, i=0, \ldots, n$, with $F_{i}$ clustering only on the $i$ th component $\partial_{i}$ of $U$, and $U_{i}$ is the simply connected component of $S \backslash \partial_{i}$ containing $U$, then Stout [S] has shown that interpolation of $C(F \cap U)$ by $\mathfrak{S}(U)$ is precisely equivalent to interpolation of $C\left(F_{i}\right)$ by $\mathfrak{S}\left(U_{i}\right)$ for all $i$, and this of course can be stated in terms of Carleson's criterion [H 2]. At least when only finitely many components of $K^{0}$ are multiply connected (so they can be neglected in showing $M$ exists) we can thus easily express (b) entirely in terms of a bound on the compositions of certain Blaschke products and Riemann mappings.

Let $K^{0} \cap F=\left\{z_{n}\right\}=\left\{z_{1}, z_{2}, \ldots\right\}$. For the proof of 4.1 we must depart from the convention of the preceding section and consider measures on $X=\partial K \cup F$ orthogonal to $A(K)$, (respectively $R(K)$ ); however $A(K)^{\perp}$ will still denote the measures on $\partial K$ orthogonal to $A(K)$. In order to identify the measures on $X=\partial K \cup F$ orthogonal to $A(K)$ we need the following simple fact: Let $v_{n}=\delta_{z_{n}}-\lambda_{z_{n}}$, so $\nu_{n} \perp A(K)$. Then the set of measures on $X=\partial K \cup F=\partial K \cup\left\{z_{n}\right\}$ orthogonal to $A(K)$ is

$$
\left\{\sum_{n=1}^{\infty} c_{n} \nu_{n}+\mu: c \in l_{1}, \mu \in A(K)^{\perp}\right\} \text {. }
$$

Indeed (4.1) is clearly orthogonal to $A(K) \mid X$ while if $\nu$ is a measure on $X$ orthogonal to $A(K)$ then $\nu_{\left(z_{1}, z_{2}, \ldots\right)}=\sum c_{n} \delta_{z_{n}}$ with $c \in l_{1}$, so that $-\sum c_{n} \nu_{n}+\nu=\sum c_{n} \lambda_{z_{n}}$ $+\nu_{\partial K}=\mu$ is a measure on $\partial K$ orthogonal to $A(K)$, so in $A(K)^{\perp}$, and $\nu=\sum c_{n} \nu_{n}+\mu$ as desired.

We shall now prove 4.1. Suppose (a) and (b) hold. We shall show $A(K)$ interpolates $C(F)$ by showing there is a constant $C$ for which

$$
\left\|\nu_{F}\right\| \leqq C\left\|\nu_{X} \backslash_{F}\right\|
$$

${ }^{(19)} A(K) \frac{1}{\lambda}=R(K)_{\lambda}^{\frac{1}{\lambda}}$ by 3.16 and 2.12 , so rational approximation follows. 
for all $\nu$ in (4.1), which is sufficient by [Gl 1, 3.2]. But $\nu=\sum_{n} c_{n} \nu_{n}+\mu=\sum_{n} c_{n} \nu_{n}+\sum_{k} \mu_{\lambda_{k}}$ where $\lambda_{k}$ is harmonic measure of a point in the $k$ th component $U_{k}$ of $K^{0}$ as earlier and $\mu_{\lambda_{k}} \perp A(K)$ by 2.6; collecting together $\mu_{\lambda_{k}}$ and all terms of $\sum c_{n} \nu_{n}$ corresponding to those $z_{n}$ lying in $U_{k}$, we express $\nu$ as a norm convergent sum of mutually singular elements of (4.1), each summand being a measure carried by the closure of one component of $K^{0}$. So it suffices to prove we have a $C$ satisfying (4.2) for all $\nu$ carried by the closure of one component $U$ of $K^{0}$, with $C$ independent of $U$.

Let $\lambda$ be the harmonic measure of some $z$ in $U ; \lambda$ is strongly dominant for $A(K)$ by hypothesis. For $\nu=\sum c_{n} \nu_{n}+\mu$, with $\mu \ll \lambda$ and $c_{n}=0$ if $z_{n} \notin U$, we have $\nu_{F}=\sum c_{n} \nu_{n F}$ $=\sum c_{n} \delta_{z_{n}}$ since $\lambda F=0$. For $\varepsilon>0$ choose $N$ so large that $\left\|\nu_{F}\right\|-\varepsilon / 2 M<\sum_{n=1}^{N}\left|c_{n}\right|$ (and therefore $\sum_{N+1}^{\infty}\left|c_{n}\right|<\varepsilon / 2 M$ as well) where $M$, our constant in (b), is at least 1 . Set

$$
f\left(z_{n}\right)=\overline{\operatorname{sgn} c_{n}}
$$

by (b) we have a $g$ in $\mathfrak{S}(U)$ with $g=f$ on $U \cap\left\{z_{n}\right\}$ and $\|g\|_{\infty} \leqq M\|f\|_{\infty}=M$. By 3.18 we have an $h$ in $H^{\infty}(A(K), \lambda)$ for which $\hat{h}=g$ on $U$ and $\|h\|_{\infty}=\|g\|_{\infty} \leqq M$. So since $\lambda$ is strongly dominant, by 2.9 we have $f_{k}$ in $A(K)$ of norm $\leqq M$ with $f_{k} \rightarrow h$ a.e. $\lambda$, so that $\hat{f}_{k} \rightarrow \hat{h}=g$ pointwise on $\left\{z_{n}\right\} \cap U$, and

$$
\hat{f}_{k}\left(z_{n}\right) \rightarrow \overline{\operatorname{sgn} c_{n}} \text {. }
$$

Thus

$$
\begin{aligned}
\left\|\nu_{F}\right\|-\varepsilon \leqq\left\|\nu_{F}\right\|-\frac{\varepsilon}{2 M}-\frac{\varepsilon}{2 M} \cdot M & \leqq \sum_{n=1}^{N}\left|c_{n}\right|-\sum_{N+1}^{\infty}\left|c_{n}\right| \cdot M \\
& \leqq\left|\nu_{F}\left(f_{n}\right)\right|+\varepsilon
\end{aligned}
$$

for $n$ sufficiently large, and since $\left.\nu\left(f_{n}\right)=0,\left\|\nu_{F}\right\|-\varepsilon \leqq\left|\nu_{X \backslash_{F}}\left(f_{n}\right)\right|+\varepsilon \leqq M \| \nu_{X}\right\rangle_{F} \|+\varepsilon$. Since $\varepsilon>0$ is arbitrary, (4.2) follows with $C=M$ independent of $U$.

To see the converse is easy. Indeed (a) holds by the argument used in the second paragraph of this section, while $A(K) \mid F=C(F)$ implies that $g \rightarrow g \mid F$ maps $A(K)$ onto $C(F)$, so by the open mapping theorem we have a constant $M$ for which, for each $f \in C(F)$ we have a $g$ in $A(K)$ with $g=f$ on $F$ and $\|g\| \leqq M\|f\|$. Thus if $f \in C(F \cap U)$ and we set $f_{N}=f$ on $U \cap\left\{z_{1}, \ldots, z_{N}\right\}$ and $=0$ elsewhere on $\left\{z_{n}\right\}$ we have $g_{N} \in A(K)$ of norm $\leqq M\|f\|$ and $g_{N}=f$ on $U \cap\left\{z_{1}, \ldots, z_{N}\right\}$. Since $g_{N}$ is a normal family in $\mathfrak{S}(U)$ we of course obtain $g \in \mathfrak{S}(U)$ with the required properties.

5. Approximation within $R(K)$. In certain situations the results of $\S \S 2$ and 3 can be used to show the density of some subalgebras of $R(K)$. For example suppose each $z$ in $K$ has a strongly dominant representing measure for $R(K)$, while $A$ is a closed subalgebra of $R(K)$ with $K$ as its spectrum and $(\operatorname{Re} A)^{\perp}=(\operatorname{Re} R(K))^{\perp}$. Then $A$ and $R(K)$ share the same set of multiplicative measures (if $\mu$ is a multiplicative on $A$ and represents $z, \mu-\lambda_{z} \in(\operatorname{Re} A)^{\perp}$ is orthogonal to $R(K)$, so $\mu$ is multiplicative on $R(K)$ ), and thus have the same Gleason parts. So if $A$ has no completely singular orthogonal measures $A=R(K)$ by 2.12 and 2.8 . 
This section is devoted to an instance in which our earlier arguments eliminate the completely singular measures. The result is an awkward relative of a result of Bishop [B 1].

THEOREM 5.1. Suppose strongly dominant measures exist for $R(K)$, harmonic measures are dominant, and $\partial K$ has plane measure zero $\left({ }^{20}\right)$. Suppose $A$ is a closed, inverse closed subalgebra of $R(K)$ with the same real orthogonal measures as $R(K)$ on $\partial K$, and contains a subset $\mathscr{G}$ whose elements do not vanish on $\partial K$ and satisfy the following:

(a) $1 \in \mathscr{G}$ and $g-c \in \mathscr{G}$ if $g \in \mathscr{G}$ and $c \in C \mid g(\partial K)$.

(b) $g \in \mathscr{G}$ is analytic near $K$.

(c) $\mathscr{G}$ separates $\partial K$.

(d) Let $g \in \mathscr{G}$. Then for each $z \in \partial K$ there is a closed neighborhood $V$ of $z$ in $K$ and an $f \in C+g A$ for which: (i) harmonic measures are dominant for $R(f(K))$ (so $\partial f(K)$ is the minimal boundary, in particular); (ii) $f$ maps $V$ homeomorphically with $V=f^{-1} f(V)$.

Then $A=R(K)$.

For the purposes of our proof we let $\lambda_{K}^{*}$ denote our measure $\lambda^{*}=\sum 2^{-n} \lambda_{n}$, where $\lambda_{n}$ is the harmonic measure of some point in the $n$th component of $K^{0} ; \lambda_{V}^{*}$ denotes the corresponding measure for $V$. Let $B=C+g A$ as in (d), and let $\mu$ be a probability measure on $\partial K$, multiplicative on $B$, and not a point mass. We shall show $\mu \ll \lambda_{R}^{*}$.

Let $z$ lie in the closed support of $\mu$, and choose $V$ and $f$ as in (d). Shrinking $V$ (so $f$ becomes an open homeomorphism of a neighborhood of $V$ in $K$ onto a neighborhood of $f(V)$ in $f(K)$ ) we can assume $V=K \cap W$, with $W$ a closed disc about $z$, and that $f$ maps $V \cap \partial K$ into $\partial f(K)$. With $V=K \cap W$, all points of $\partial V$ are regular [T], and the same is true for $\partial f(V)$ : for $f$ is conformal on $V \mid(V \cap \partial K)$, and $V \cap \partial K$ maps into $\partial f(K)$ which is the minimal boundary for $R(f(K))$ so that barriers exist for all points in $\partial f(V)$.

We need the fact that on the relatively open subset $E=V \cap \partial K \backslash \partial W=\partial V \backslash \partial W$ of $\partial V$ we have $\lambda_{K}^{*} \ll \lambda_{V}^{*}$ (and the corresponding fact for $f(K), f(V)$ and $f(E)=$ $\partial f(V) \backslash f(\partial W)$ ), and this follows from the argument used to prove 3.9, which we briefly recapitulate (omitting the passage from continuous to discontinuous boundary values, as given in 3.9 ).

If $F$ is a compact subset of $E$ and $\lambda_{V}^{*} F=0$, setting

$$
\begin{aligned}
f & =0 \text { on } E, \\
& =\lambda_{z} F \text { on } V \backslash E,
\end{aligned}
$$

we have $f \in C(\partial V)$, and the harmonic function $w \rightarrow \lambda_{w} F-\hat{f}(w)$ on $V^{0}$ (where $\hat{f}$ is the continuous harmonic extension of $f$ to $V$ ) has boundary values $=\chi_{F}$ on $E$,

$\left({ }^{20}\right)$ This condition and (b) below are chosen to insure that $R(g(\partial K))=C(g(\partial K))$ for any $g \in \mathscr{G}$; any other conditions insuring this would do as well. 
and $=0$ on $\partial V \backslash E$, and so coincides with $w \rightarrow \lambda_{w}^{V}(F)$, which vanishes on $V^{0}$ since $\lambda_{V}^{*} F=0$. Thus $\lambda_{w} F=\hat{f}(w)$ on $V^{0}$, and since $\hat{f} \rightarrow 0$ as we approach $F$ in $V^{0}$, hence in $K^{0}$, while $\lambda_{w} F \rightarrow 0$ as we approach $\partial K^{0}$ in $K^{0}$ away from $F$, we see the harmonic function $w \rightarrow \lambda_{w} F$ vanishes on $K^{0}$, so that $\lambda_{K}^{*} F=0$ as desired. Similarly $\lambda_{f(K)}^{*} \ll \lambda_{f(V)}^{*}$ on $\partial f(V) \backslash f(\partial W)$.

Now let $f^{*}$ denote the map of measures on $\partial K$ into measures on $f(K)$ dual to the map $g \rightarrow g \circ f$ of $C(f(K))$ into $C(\partial K)$. Then since $A$ is inverse closed, for any rational function $g$ without poles in $f(K)$ we have $g \circ f \in A$ (if $p$ is a polynomial without zeroes on $f(K), p \circ f \in A$ does not vanish on $K$, so $1 /(p \circ f) \stackrel{\circ}{=}(1 / p) \circ f \in A)$. So $g \circ f \in A$ for $g \in R(f(K))$, and thus $g \rightarrow f^{*} \mu(g)=\mu(g \circ f)$ is multiplicative on $R(f(K))$. Moreover $f^{*} \mu$, which has $f(z)$ in its closed support, is not a point mass unless it represents $f(z)$, and then $\mu$ would necessarily be $\delta_{z}$ since $f$ is a homeomorphism on $V$ and $V=f^{-1} f(V)$.

Thus, swept to $\partial f(K), f^{*} \mu$ yields a non-point-mass multiplicative measure on $R(f(K))$ which is $\geqq\left(f^{*} \mu\right)_{f(V)}=f^{*}\left(\mu_{V}\right)$ since the support of $\mu_{V}$, which lies in $V \cap \partial K$, maps into $\partial f(K)$. Since the swept measure must represent a point in $f(K)^{0}$ by (d), $\left(f^{*} \mu\right)_{f(V)} \ll \lambda_{f(K)}^{*}$, again by (d). As noted $\lambda_{f(K)}^{*} \ll \lambda_{f(V)}^{*}$ on $\partial f(V) \backslash f(\partial W)$, hence on $f\left(K \cap W^{0}\right)$ so that $f^{*} \mu \ll \lambda_{f(V)}^{*}$ on $f\left(K \cap W^{0}\right)$, which implies $\mu \ll \lambda_{V}^{*}$ on $K \cap W^{0}$. But $\lambda_{V}^{*} \ll \lambda_{K}^{*}$ on $K \cap W^{0}$, as one sees by sweeping, so $\mu_{K} \cap w^{0} \ll \lambda_{K}^{*}$. Since $z$ is an arbitrary point in the support of $\mu, \mu \ll \lambda_{K}^{*}$.

In particular for the element 1 of $B$ we see $B=C+1 A=A$ has any (nondiscrete) multiplicative measure $\mu \ll \lambda_{K}^{*}$, so $\mu$ represents a point in $\mathfrak{M}_{A}$ lying in the same Gleason part as some $z$ in $K^{0}$. But $\mathrm{M}_{z}(A)=\mathrm{M}_{z}(R(K))$ since if $\nu \in \mathrm{M}_{z}(A), \nu-\lambda_{z}$ $\in(\operatorname{Re} A)^{\perp}=(\operatorname{Re} R(K))^{\perp}$ implies $\nu \in \mathrm{M}_{z}(R(K))$. Thus for each part $P$ for $A$ we have an element $z$ of $K$ in $P$ with a strongly dominant representing measure $\lambda$, equivalent to $\lambda_{z}$ since harmonic measures are dominant for $R(K)$ by hypothesis. Since $(\operatorname{Re} A)^{\perp}$ $=(\operatorname{Re} R(K))^{\perp}, 2.12$ shows $A_{\lambda}^{\perp}=R(K)_{\lambda}^{\perp}$, and thus in the decomposition of $A^{\perp}$ and of $R(K)^{\perp}$, given by $2.8, A^{\perp}$ and $R(K)^{\perp}$ can only differ in the completely singular components: so to see $A=R(K)$ it suffices to show $S=0$, where $S$ is the set of completely singular measures orthogonal to $A$. (The corresponding measures for $R(K)$ are included in $S$.)

Now let $B_{0}=\{g \in C(\partial K): g \perp S\} . B_{0}$ is a (closed) subalgebra of $C(\partial K)$ since $g \in B_{0}, \sigma \in S$ imply $g \perp f \sigma$ for $f \in A$, or $g \sigma \perp f$; so $g \sigma \perp A$, and being singular, $g \sigma \in S$. Thus $h g \sigma \in S$ for $h \in B_{0}$, and $\sigma(h g)=g h \sigma(1)=0$, so $h g \in B_{0}$, and $B_{0}$ is an algebra. Of course $A$ is a subalgebra, and we shall next see each $g \in \mathscr{G}$ has its spectrum in $B_{0}$ precisely $g(\partial K)$.

The spectrum of $g$ in $A$ is contained in $g(K)$ as we already know, since $A$ is inverse closed. So the spectrum of $g$ in $B_{0}$ lies in $g(K)$. For $t=g(z) \in g(K), z \in K$, $t \notin g(\partial K)$, we have $g_{1}=g-t$ an invertible in $C(\partial K)$ which lies in $\mathscr{G}$. By our earlier argument we know each measure $\mu$ which is multiplicative on $B=C+g_{1} A$ and not a point-mass is $\ll \lambda_{R}^{*}$. Thus dominant measures exist for $B$.

Now let $\sigma \in S$, so that $\sigma \perp B$, and, since $|\sigma|$ and $\lambda_{K}^{*}$ are mutually singular, $\sigma$ is a 
completely singular orthogonal measure for $B$. For our $z \in K$ with $g(z)=t$ we have $g_{1}(z)=0$, so

$$
\left(1 / g_{1}\right) \sigma-\sigma\left(1 / g_{1}\right) \cdot \lambda_{z}
$$

is orthogonal to $C+g_{1} \cdot A=B$; by $2.6,\left(1 / g_{1}\right) \sigma$, its singular component relative to the dominant measure for $z$ on $B$, is also orthogonal to $B$. So $\sigma\left(1 / g_{1}\right)=\left(1 / g_{1}\right) \sigma(1)=0$. Since this holds for all $\sigma \in S$ we have $1 / g_{1} \in B_{0}$, so that the spectrum of $g \in \mathscr{G}$ in $B_{0}$ is $g(\partial K)$ as asserted. Hence $h \circ g \in B_{0}$ for each $h \in R(g(\partial K))$.

But $g \in \mathscr{G}$ is analytic near $K$ and $\partial K$ has plane measure zero, so that $g(\partial K)$ also has plane measure zero, and $R(g(\partial K))=C(g(\partial K))$ by the well-known result of Hartogs and Rosenthal (which is contained in the familiar result of Bishop [B 2]), and thus $h \circ g B_{0}$ for each $h \in C(g(\partial K))$, which in particular says $\bar{g} \in B_{0}$. Since the elements of $\mathscr{G}$ separate $\partial K$, we have $B_{0}=C(\partial K)$ by Stone-Weierstrass, and $S=0$ as desired, completing our proof.

\section{REFERENCES}

[A] P. R. Ahern, On the generalized F. and M. Riesz theorem, Pacific J. Math. 15 (1965), 373-376.

[AS 1] P. R. Ahern and Donald Sarason, The $H^{p}$ spaces of a class of function algebras, Acta Math. 117 (1967), 123-163.

[AS 2] - On some hypodirichlet algebras of analytic functions, Amer. J. Math. (to appear).

[Ar] R. Arens, The maximal ideals of certain function algebras, Pacific J. Math. 8 (1958), 641-648.

[B 1] E. Bishop, Subalgebras of functions on a Riemann surface, Pacific J. Math. 8 (1958), 29-50.

[B 2] —, A minimal boundary for function algebras, Pacific J. Math. 9 (1959), 629-642.

[B 3] — Boundary measures of analytic differentials, Duke Math. J. 27 (1960), 331-340.

[BW] A. Browder and J. Wermer, Some algebras of functions on an arc, J. Math. Mech. 12 (1963), 119-130.

[C] L. Carleson, Mergelyan's theorem on uniform polynomial approximation, Math. Scand. 15 (1964), 167-175.

[D] M. M. Day, Normed linear spaces, Springer, Berlin, 1958.

[DS] N. Dunford and J. T. Schwartz, Linear operators. I, Interscience, New York, 1958.

[F] F. Forelli, Analytic measures, Pacific J. Math. 13 (1963), 571-578.

[G] A. Gleason, Function algebras, Seminar on Analytic Functions, Vol. II, Institute for Advanced Study, Princeton, N. J., 1957, 213-226.

[GaGl] J. Garnett and I. Glicksberg, Algebras with the same multiplicative measures, J. Functional Anal. (to appear).

[Gl 1] I. Glicksberg, Measures orthogonal to algebras and sets of antisymmetry, Trans. Amer. Math. Soc. 105 (1962), 415-435.

[Gl 2] — Multiplicative functionals with norm separable sets of representing measures, Function Algebras (Proc. of Tulane Univ. Sympos.), Scott Foresman, Chicago, 1965.

[Gl 3] — The abstract F. and M. Riesz theorem, J. Functional Anal. 1 (1967), 109-122.

[GIW] I. Glicksberg and J. Wermer, Measures orthogonal to a dirichlet algebra, Duke Math. J. 30 (1963), 661-666. 
[H 1] K. Hoffman, Analytic functions and logmodular Banach algebras, Acta Math. 108 (1962), 271-317.

[H 2] —, Banach spaces of analytic functions, Prentice-Hall, Englewood Cliffs, N. J., 1962.

[K] B. Kerekjarto, Topologie. I, Springer, Berlin, 1923.

[L] G. Lumer, Analytic functions and dirichlet problem, Bull. Amer. Math. Soc. 70 (1964), 98-104.

[MM] M. Marcus and H. Minc, A survey of matrix theory and matrix inequalities, Allyn and Bacon, Boston, 1964.

[M] S. N. Mergelyan, Uniform approximations to functions of a complex variable, Amer. Math. Soc. Transl. (1) 3 (1962), 294-391.

[Ne] Z. Nehari, Conformal mapping, McGraw-Hill, New York, 1952.

[N] M. H. A. Newman, Topology of plane sets of points, Cambridge Univ. Press, Cambridge, 1939.

[RN] F. Riesz and B. Sz.-Nagy, Leçons d'analyse fonctionnelle, Akadémiai Kiadó, Budapest, 1955.

[S] E. L. Stout, Bounded holomorphic functions on finite Riemann surfaces, Trans. Amer. Math. Soc. 120 (1965), 255-285.

[T] M. Tsuji, Potential theory in modern function theory, Maruzen, Tokyo, 1959.

[Wa 1] J. L. Walsh, Über die Entwicklung einer harmonischer Funktion nach harmonischen Polynomen, J. Reine Angew. Math. 159 (1928), 197-209.

[Wa 2] - The approximation of harmonic functions by harmonic polynomials and harmonic rational functions, Bull. Amer. Math. Soc. 35 (1929), 499-544.

[We 1] J. Wermer, Polynomial approximation on an arc in $C^{3}$, Ann. of Math. 62 (1955), 269-270.

[We 2] —, Dirichlet algebras, Duke Math. J. 27 (1960), 373-382.

[We 3] - Banach algebras and analytic functions, Advances in Math., Vol. 1, Academic Press, New York, 1961.

[We 4] — Analytic discs in maximal ideal spaces, Amer. J. Math. 86 (1964), 161-170.

[We 5] - Seminar über Funktionen-Algebren, Springer, Berlin, 1964.

[Wi] D. R. Wilken, Lebesgue measure of parts for $R(X)$, Proc. Amer. Math. Soc. 18 (1967), 508-512.

\section{UNIVERSITY OF WASHINGTON, SeATtLe, Washington}

